\title{
The CPLEAR Electromagnetic Calorimeter ${ }^{1}$
}

\author{
R. Adler ${ }^{1}$, G. Backenstoss ${ }^{1}$, F. Bal ${ }^{2}$, O. Behnke ${ }^{5}$, P. Bloch $^{2}$, D. Damianoglou ${ }^{4}$, \\ P. Dechelette ${ }^{2}$, M. Dröge ${ }^{5}$, B. Eckart ${ }^{1}$, C. Felder ${ }^{1}$, W. Fetscher ${ }^{5}$, M. Fidecaro ${ }^{2}$, \\ D. Garreta ${ }^{2}$ 2, H.-J. Gerber ${ }^{5}$, P. Gumplinger ${ }^{5}$, D. Guyon ${ }^{2}$, H.U. Johner ${ }^{3}$, B. Lofstedt ${ }^{2}$, \\ J. Kern ${ }^{3}$, P. Kokkas ${ }^{1}$, H. Krause ${ }^{1}$, U. Mall ${ }^{1}$, C. Marin ${ }^{2}$, F. Nanni ${ }^{2}$, B. Pagels ${ }^{5}$, \\ P. Pavlopoulos ${ }^{1}$, P. Petit ${ }^{2}$, G. Polivka ${ }^{1}$, Ch. Rheme ${ }^{3}$, T. Ruf ${ }^{2}$, C. Santoni ${ }^{1}$, \\ L.A. Schaller ${ }^{3}$, A. Schopper ${ }^{2}$, L. Tauscher ${ }^{1}$, H. Tschopp ${ }^{3}$, P. Weber $^{5}$, H. Wendler ${ }^{2}$, \\ C. Witzig ${ }^{5}$, M. Wolter ${ }^{5}$. \\ ${ }^{1}$ University of Basle, CH-4056 Basle, Switzerland \\ ${ }^{2}$ CERN, CH-1211 Geneva 23, Switzerland \\ ${ }^{3}$ University of Fribourg, CH-1700 Fribourg, Switzerland \\ ${ }^{4}$ University of Thessaloniki, GR-54006 Thessaloniki, Greece \\ ${ }^{5}$ ETH-IPP Zürich, CH-8093 Zürich, Switzerland
}

\begin{abstract}
A large-acceptance lead/gas sampling electromagnetic calorimeter (ECAL) was constructed for the CPLEAR experiment to detect photons from decays of $\pi^{0} \mathrm{~s}$ with momentum $p_{\pi^{0}} \leq 800 \mathrm{MeV} / c$. The main purpose of the ECAL is to determine the decay vertex of neutral-kaon decays $\mathrm{K}^{0} \rightarrow \pi^{0} \pi^{0} \rightarrow 4 \gamma$ and $\mathrm{K}^{0} \rightarrow \pi^{0} \pi^{0} \pi^{0} \rightarrow 6 \gamma$. This requires a positionsensitive photon detector with high spatial granularity in $r-, \varphi$-, and $z$-coordinates. The ECAL - a barrel without end-caps located inside a magnetic field of $0.44 \mathrm{~T}$ - consists of 18 identical concentric layers. Each layer of $1 / 3$ radiation length $\left(\mathrm{X}_{0}\right)$ contains a converter plate followed by small cross-section high-gain tubes of $2640 \mathrm{~mm}$ active length which are sandwiched by passive pick-up strip plates. The ECAL, with a total of $6 \mathrm{X}_{0}$, has an energy resolution of $\sigma(E) / E \approx 13 \% / \sqrt{E(\mathrm{GeV})}$ and a position resolution of $4.5 \mathrm{~mm}$ for the shower foot. The shower topology allows separation of electrons from pions. The design, construction, read-out electronics, and performance of the detector are described.

(submitted to Nuclear Instruments and Methods A)

\footnotetext{
${ }^{1}$ The CPLEAR collaboration: University of Athens, University of Basle, Boston University, CERN, LIP and University of Coimbra, Delft University of Technology, University of Fribourg, University of Ioannina, University of Liverpool, J. Stefan Inst. and Phys. Dep., University of Ljubljana, CPPM, IN2P3-CNRS et Université d'Aix-Marseille II, CSNSM, IN2P3-CNRS, Paul-Scherrer-Institut(PSI), DAPNIA/SPP, CE Saclay, Royal Institute of Technology, University of Thessaloniki, ETH-IPP Zürich.
}

${ }^{2}$ On leave from DAPNIA/SPP, CE Saclay, France.
\end{abstract}




\section{Introduction}

The CPLEAR experiment investigates CP, T, and CPT symmetries in the neutral-kaon system [1-5] at the Low-Energy Antiproton Ring (LEAR), CERN. The neutral kaons ${ }^{3}$, $\mathrm{K}^{0}$, are produced and strangeness-tagged in $\overline{\mathrm{p}} \mathrm{p}$-annihilation at rest, i.e. $\overline{\mathrm{p}} \mathrm{p} \rightarrow \mathrm{K}^{+} \pi^{-} \overline{\mathrm{K}}^{0}$ and $\overline{\mathrm{p}} \mathrm{p} \rightarrow \mathrm{K}^{-} \pi^{+} \mathrm{K}^{0}$. The study includes final states with neutral pions, i.e. $\mathrm{K}^{0} \rightarrow 2 \pi^{0}$ $[5], \rightarrow 3 \pi^{0}[6], \rightarrow \pi^{+} \pi^{-} \pi^{0}[3,4]$, emitting photons mainly in the 50-500 MeV energy range, and semileptonic final states with $e^{ \pm}$and $\pi^{\mp}$. To cope with these decay channels, though predominantly with $\mathrm{K}^{0} \rightarrow 2 \pi^{0}$, a position-sensitive detector for low-energy photons, ECAL (Electromagnetic CALorimeter), was constructed as a barrel without end-caps which fits in between the cylindrical geometry of the inner detector parts and the solenoid magnet of the CPLEAR set-up. The calorimeter - subdivided into three azimuthal sectors - covers $85 \%$ of the solid angle, and consists of 18 identical concentric layers. Each layer is equivalent to $1 / 3$ radiation length $\left(\mathrm{X}_{0}\right)$ and contains:

- A converter plate (1.5 mm lead).

- A layer of small-size high-gain tubes [7]. Each tube is instrumented with a wire (W) of $2640 \mathrm{~mm}$ active length, providing $r$ and $\varphi^{4}$ information. The tubes are packed in 'chambers' by groups of eight.

- Two layers of pick-up strips, one situated below (U strips) and one above (V strips) the chambers, in close contact with them. The $\mathrm{U}$ and $\mathrm{V}$ strips are positioned at angles of $+30^{\circ}$ and $-30^{\circ}$ to the tube-wire axis, respectively, allowing the hit coordinates along the wires ( $z$ information) to be determined unambiguously for every layer.

This paper is outlined as follows: The design and construction of the ECAL are reported in Section 2. The production of the high-gain tubes is described in Section 3 and Appendix A, and the read-out electronics in Section 4. The performance of ECAL is presented in Section 5, while a description of the Monte Carlo (MC) simulation is given in Appendix B.

\section{Design and Construction of the ECAL}

A detailed description of the CPLEAR experimental set-up is given in Ref. [8]. A side view of the whole CPLEAR detector parallel to the beam line is given in Fig. 1, while a transverse view of the active detector region is displayed in Fig. 2.

From the very beginning, the design of the ECAL was geometrically constrained by both the inner and the outer radii. The study of the $\mathrm{K}_{\mathrm{S}} \mathrm{K}_{\mathrm{L}}$ interference in the $\mathrm{K}^{0} \rightarrow 2 \pi$ channels requires a $\mathrm{K}^{0}$ decay length corresponding to an eigentime $\tau$ of up to about $20 \tau_{S}\left(\tau_{S}\right.$ is the $\mathrm{K}_{\mathrm{S}}$ mean life), equivalent to $60 \mathrm{~cm}$ at our momenta. Tracking devices (proportional chambers, drift chambers, and streamer tubes) surrounded by a $12.4 \mathrm{~cm}$

\footnotetext{
${ }^{3}$ Unless explicitly stated, we mean by $\mathrm{K}^{0}$ either $\mathrm{K}^{0}$ or $\overline{\mathrm{K}}^{0}$.

${ }^{4}$ We use a cylindrical coordinate system with the origin at the center of the magnet and the $z$-axis defined along the incoming antiproton beam, while $r$ and $\varphi$ span the transverse plane. Hits in the calorimeter are measured in auxiliary coordinates $\mathrm{W}, \mathrm{U}$, and $\mathrm{V}$. W corresponds directly to $r$ and $\varphi$, while combinations of U-W and $\mathrm{V}-\mathrm{W}$ provide $z$.
} 


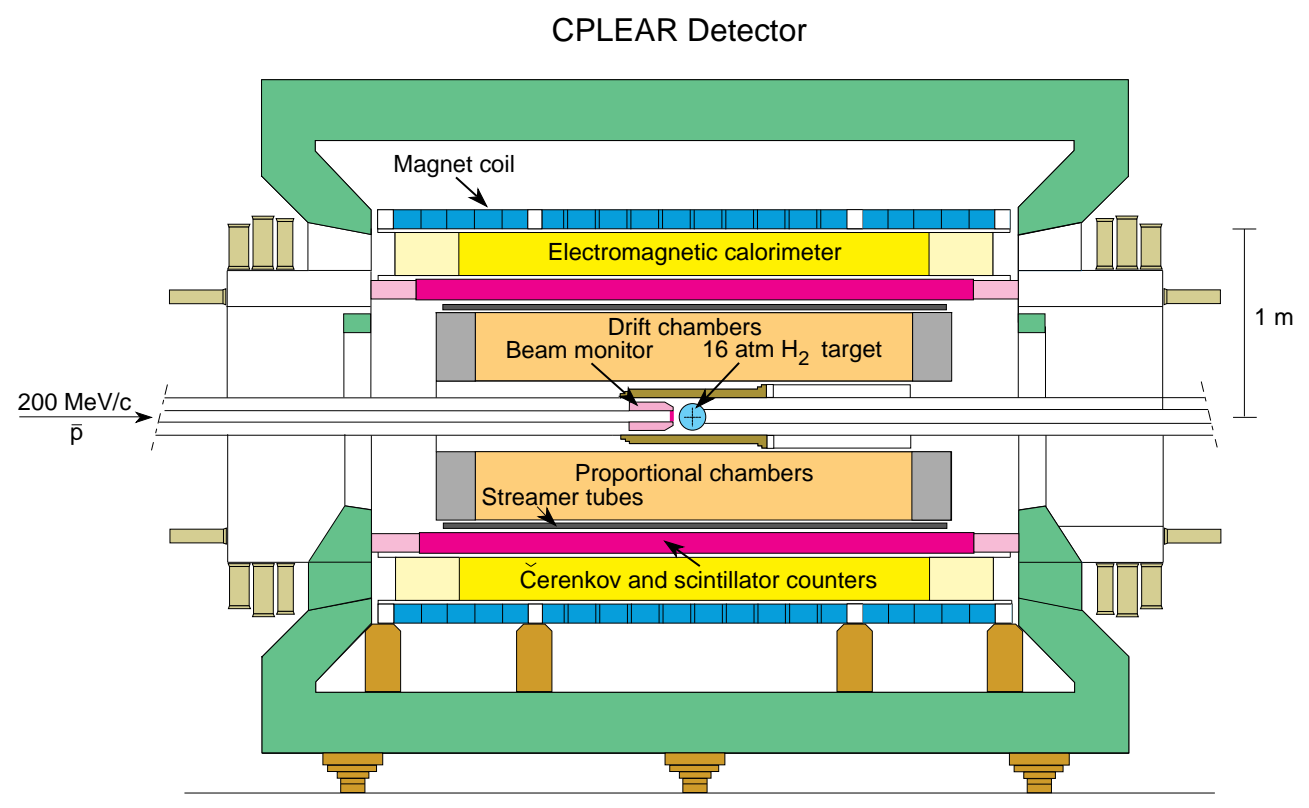

Figure 1: Side view of the CPLEAR detector layout parallel to the beam line ( $z$ axis).
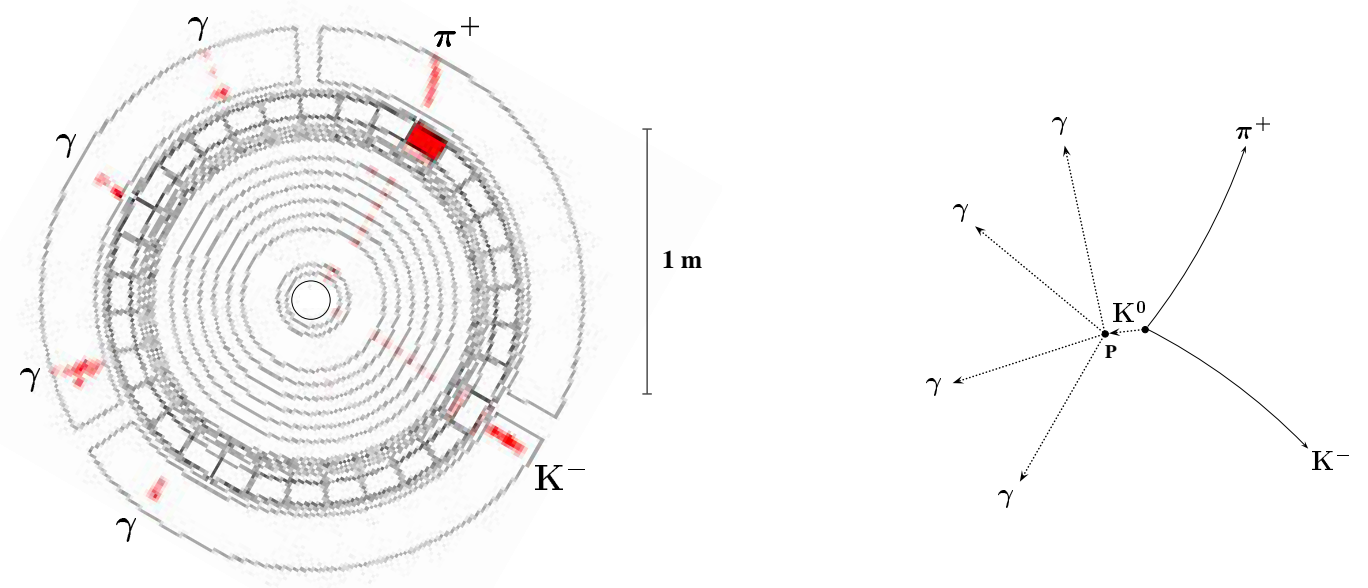

Figure 2: Transverse $(r, \varphi)$ view of the CPLEAR detector showing the hit pattern (left) and the reconstruction (right) of a $\bar{p} p \rightarrow \pi^{+} \mathrm{K}^{-} \mathrm{K}^{0}$ annihilation event with subsequent $\mathrm{K}^{0} \rightarrow 2 \pi^{0} \rightarrow 4 \gamma$ decay. 
thick particle identification detector, PID (Cherenkov and scintillator counters), detect the charged decay products from the appropriate $\mathrm{K}^{0}$ decay volume and set a minimal inner radius of $75 \mathrm{~cm}$ for the calorimeter. On the outer side, the ECAL had to fit within an existing solenoidal magnet coil of $1 \mathrm{~m}$ radius (see Fig.1), which had been recovered from the DM2 $\mathrm{e}^{+} \mathrm{e}^{-}$experiment at Orsay. Because of the photon's low energy, a good photon detection efficiency and spatial resolution could only be achieved with the ECAL situated inside the magnet coil, which required a moderate calorimeter thickness in terms of radiation lengths. This was, however, considered sufficient for the purposes of the CPLEAR experiment.

The design criteria for the calorimeter are dictated by the ability to reconstruct as accurately as possible the $\mathrm{K}^{0} \rightarrow \pi^{0} \pi^{0} \rightarrow 4 \gamma$ decay vertex. The decay vertex resolution is a function of the $\mathrm{K}^{0}$ momentum (calculated by momentum conservation at the $\bar{p}$ annihilation vertex) and the momenta of the four photons. The design studies have shown that the spatial resolution when measuring the coordinates of $\gamma$ conversions in the detector is the most important parameter for the $\mathrm{K}^{0}$ decay vertex determination (Fig. 1 in Ref. [9]). The energy resolution plays only a smaller role for the $\mathrm{K}^{0}$ vertex position and can even be fairly poor provided the spatial resolution is better than $5 \mathrm{~mm}$. The energy information is however useful for the pairing of photons to $\pi^{0} \mathrm{~s}$ and background rejection. The simulations have also shown that the best spatial resolution of the conversion point of low-energy photons is obtained by sampling the shower as close as possible to the $\gamma$ conversion point. Therefore, the calorimeter demands high granularity in $r, \varphi$, and $z$ to measure the shower foot, i.e. the location of the beginning of the shower development.

The choice of geometry and material for the ECAL is the result of a compromise between the geometrical constraints, optimization of performance, and cost. All these considerations lead us to exclude the choice of a crystal calorimeter (such as CsI), which would have been very costly. Instead, we have opted for a lead/gas sampling calorimeter with a fine sampling fraction of $1 / 3 \mathrm{X}_{0}$. As an active medium, we use high-gain tubes of small cross-section for the $r$ - and $\varphi$-coordinates, allowing the measurement of the $z$ coordinate along the tube wires by means of pick-up strips. Our design permits accurate measurement of the shower foot, achieved by the spatial matching of the W (wire), $\mathrm{U}$ and $\mathrm{V}$ (strip) information, also for $\gamma$ 's impinging at large incidence angle with respect to the calorimeter inner surface. This is in contrast to a possible design with crystals, where the photon average incidence angle of $\approx 30^{\circ}$ for $\mathrm{K}^{0} \rightarrow 2 \pi^{0} \rightarrow 4 \gamma$ events would introduce a position uncertainty of half a radiation length resulting from the projection of the shower barycentre. Digital read-out of the ECAL was selected as suggested by MC simulation studies. These studies showed that the best energy resolution for photons of only a few hundred $\mathrm{MeV}$ energy to be detected in a sampling calorimeter is obtained by counting the number of hits in the track segments rather than measuring the energy deposited in the gas. Thus, measurement of the entire shower development allows the number of $\mathrm{W}$ hits (tubes fired) to be related to the shower energy. Information on the shower direction is also obtained from measurement of the entire shower.

The large weight and complexity of the read-out electronics and the cabling suggested an azimuthal segmentation of ECAL (a longitudinal segmentation would have been impossible). However, the need for every pick-up strip to reach either the upstream or downstream end of the detector puts a strong constraint on the azimuthal segmentation. The maximum number of azimuthal sectors is given by $n=\operatorname{Int}(2 \pi R /(L \operatorname{tg} \theta))$, where $R$ is the inner radius, $L$ the active strip length, and $\theta$ the strip angle relative to the cylinder 
axis. For the chosen value of $\theta=30^{\circ}$, the number of sectors can only be $n \leq 3$. The ECAL was developed with three azimuthal sectors.

The ECAL represents a total of $6 \mathrm{X}_{0}$ and is $20 \mathrm{~cm}$ thick (18 layers). For layers L2 to L18, the radiation length is $1 / 3 \mathrm{X}_{0}$ and the thickness is $11.25 \mathrm{~mm}$ per layer. A transverse cross-section of the calorimeter is shown in Fig. 3. In layer L1, the aluminium of the

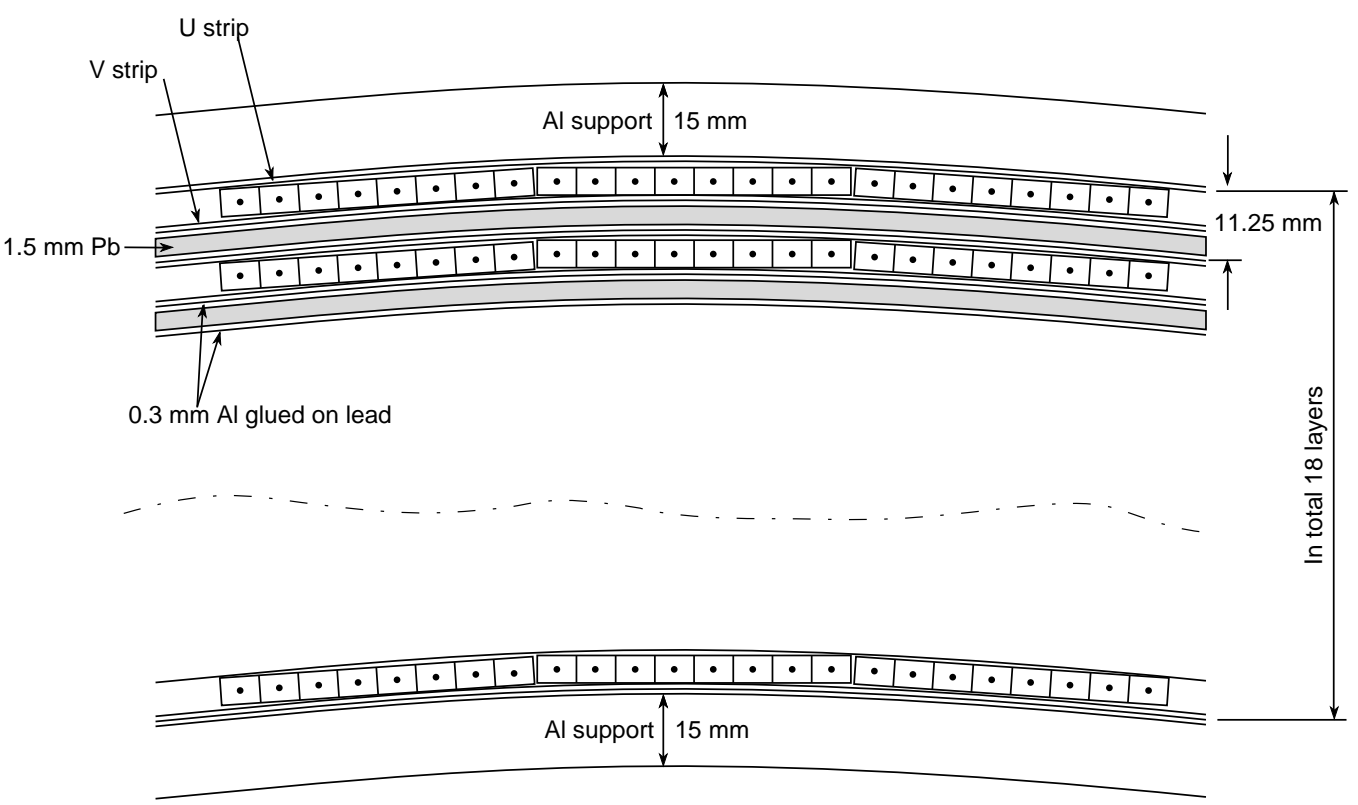

Figure 3: Transverse cross-section of the calorimeter.

inner cylinder $(15 \mathrm{~mm})$ acts as a converter $\left(0.17 \mathrm{X}_{0}\right)$, i.e. no lead was placed between the cylinder and the copper strip of layer L1. The particle identification detector in front of the ECAL also acts as a converter $\left(0.54 \mathrm{X}_{0}\right)$. See Table 1 for detailed information on thicknesses and radiation lengths.

\subsection{Mechanical structure}

The total weight of the ECAL is about 8 tons which requires the support structure shown in Fig. 4. The calorimeter was assembled between two coaxial aluminium cylinders, each $15 \mathrm{~mm}$ thick and $3340 \mathrm{~mm}$ long (inner radius of the inner cylinder was $76 \mathrm{~cm}$; outer radius of the outer cylinder was $99 \mathrm{~cm}$ ), connected by three $20 \mathrm{~mm}$ thick, $3340 \mathrm{~mm}$ long aluminium bars at azimuthal angles of $0^{\circ}, 120^{\circ}$ and $240^{\circ}$ to form three sectors T1-T3 (see Fig. 4a). The bars are made of 18 subpieces of $11.25 \mathrm{~mm}$ thickness, forming a comb (see Fig. 4b) in which the lead plates are glued. Therefore, the plates are only secured at the edges. Two bolts attach the bars to the inner and outer cylinders and reinforce their stiffness. The width of the converter plates was carefully adjusted to the theoretical length, so that for each layer they follow an arc of the cylinder on which the strips and chambers are located.

Before insertion of the chambers, the mechanical structure was relatively flexible. Such a design could only be adopted because the chambers themselves have a very good mechanical rigidity (see below). It offers the advantage of being extremely compact, a property required by the physical space limitations, but the disadvantage of not being 
Table 1: Materials, thicknesses, and corresponding radiation lengths. The converter is listed separately for layer L1 and layers L2 to L18. Average thicknesses are used for computing the tube components (numbers in parentheses are averages). Pure copper is assumed for the brass wings.

\begin{tabular}{|c|c|c|c|}
\hline Detector element & Material & Thickness & Radiation length \\
\hline \hline & & {$[\mathrm{mm}]$} & {$\left[\mathrm{X}_{0}\right]$} \\
\hline \hline Converter & Al (L1) & 15.0 & 0.1685 \\
& $\mathrm{Al}(\mathrm{L} 2-\mathrm{L} 18)$ & $2 \times 0.3$ & $2 \times 0.0034$ \\
& $\mathrm{~Pb}(\mathrm{~L} 2-\mathrm{L} 18)$ & 1.5 & 0.2679 \\
\hline U,V-strip & $\mathrm{Cu}$ & $2 \times 0.035$ & $2 \times 0.0024$ \\
& $\mathrm{G} 10$ & 0.6 & 0.0031 \\
\cline { 3 - 4 } & & 0.67 & 0.0080 \\
\hline Chamber & G10 & $2 \times 1.0$ & $2 \times 0.0052$ \\
(8 tubes) & $\mathrm{Cu}($ wings) & $(0.53)$ & $(0.0370)$ \\
& $\mathrm{W}$ (wire) & $(0.0002)$ & $\left(6.8 \cdot 10^{-5}\right)$ \\
& gas & $(3.39)$ & $\left(2.0 \cdot 10^{-5}\right)$ \\
\cline { 3 - 4 } & & 6.0 & $(0.0475)$ \\
\hline \hline L1 & & 26.25 & 0.2320 \\
L2-L18 & & 11.25 & $0.3382(\mathrm{per}$ layer $)$ \\
\cline { 3 - 4 } Total & & & 6.0 \\
\hline
\end{tabular}
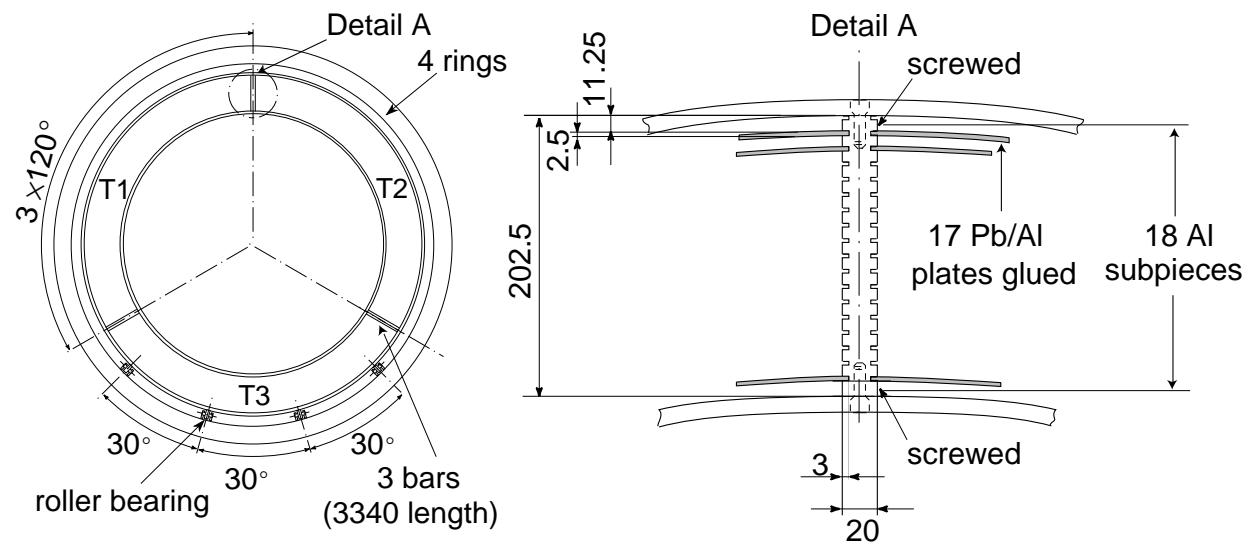

a)

b)

Figure 4: a) Transverse cross-section of the mechanical support structure. b) Detail of the bar between two sectors. All dimensions in mm. 
easy to handle. The weight and the large number of detector components rendered it very difficult to test and calibrate the ECAL in a test beam before the final installation.

Once the ECAL was ready for installation, it was slid inside the magnetic coils, which are interleaved with 4 aluminium support rings, each $100 \mathrm{~mm}$ wide (Figs. 1 and 4 ).

\subsection{High-gain tubes}

The design and performance of the high-gain tubes have already been published elsewhere $[7,9]$. Some of their properties are recalled here for completeness.

The $4 \times 4.5 \mathrm{~mm}^{2}$ cross-section tubes (Fig. 5), operated in a mode very near to the limited-streamer mode, are built in the form of gas-tight packages of eight separate tubes (Fig. 5a), referred to as chambers, through which a gold-plated $40 \mu \mathrm{m}$ tungsten-rhenium wire ${ }^{5}$ is stretched.

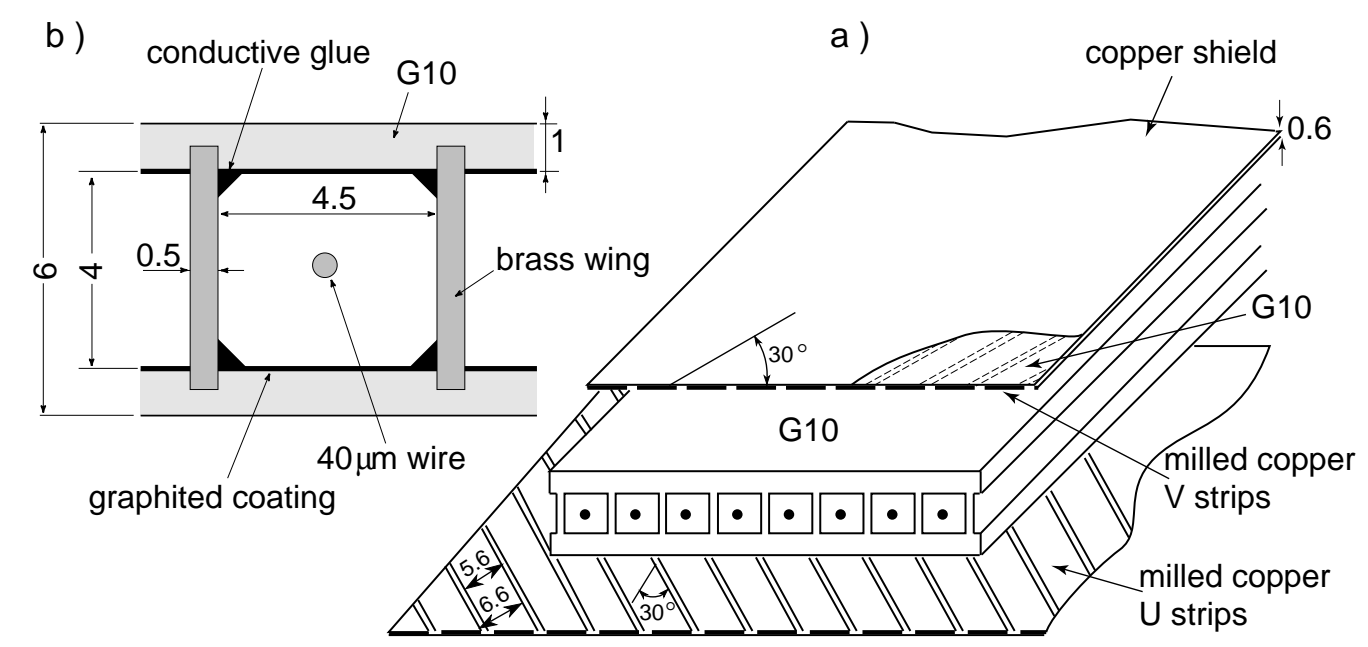

Figure 5: a) Chamber layout with 8 high-gain tubes. The strip plates above (V) and below (U) the chamber are also shown. b) Cross-section of one tube. (All dimensions are in mm, unless otherwise stated)

The chambers have a $6 \times 42.5 \mathrm{~mm}^{2}$ cross-section and $2640 \mathrm{~mm}$ active length. The calorimeter contains 40-45 chambers per layer and per sector, giving a total of 2142 chambers (17 112 tubes or wires).

Each chamber package (Fig. 5) is made of two $42.5 \mathrm{~mm}$ wide $\mathrm{G} 10^{6}$ plates (top and bottom covers) linked by nine $5 \mathrm{~mm}$ high, $0.5 \mathrm{~mm}$ thick brass wings (side walls). Both covers are coated on the gas side with a graphited paint to facilitate the grounding of the ions.

\footnotetext{
${ }^{5}$ Luma Metall, Lumalampan AB, Kalmar, Sweden. (Plated weight: 23-25 mg/m, 3-5\% Au, 861/60 W alloy with $3 \% \mathrm{Re})$

${ }^{6}$ G10 (NEMA): STESALIT 4412 FG, Stesalit AG, Zullwil, Switzerland. Composition: $62 \%$ fibreglass, $38 \%$ epoxide resin. Dielectric constant: $\epsilon=5$ at $1 \mathrm{MHz}$, resistivity: $\rho=10^{14} \Omega \cdot \mathrm{cm}$, density: $d=1.8$ $\mathrm{g} / \mathrm{cm}^{3}$.
} 
Copper pick-up strips are in close contact with the covers. The high resistivity of the graphite film (1 M $\Omega / \square)$ and the G10 plates makes them transparent for pulsed electric fields and thus allows the measurement of induced signals. Charge collection measurements were carried out to evaluate the material of the covers and the side walls [7]. The charge collection on the strips was found to increase with the resistivity of the covers (Fig. 8 in [7]). Metallic wings which are not transparent for electric fields were chosen in preference to graphited epoxy walls because of their superior lateral charge localization in the strips (Fig. 9 in [7]). A reduced charge collection (by a factor of 3) on the strips poses a drawback of the metallic wings, but the stability and rigidity of the chambers with metallic wings is very good.

Each tube wire is soldered on either side to a printed-circuit board (Fig. 6) and supported by seven equally-spaced plastic bridges (see Appendix A). The eight wires of a

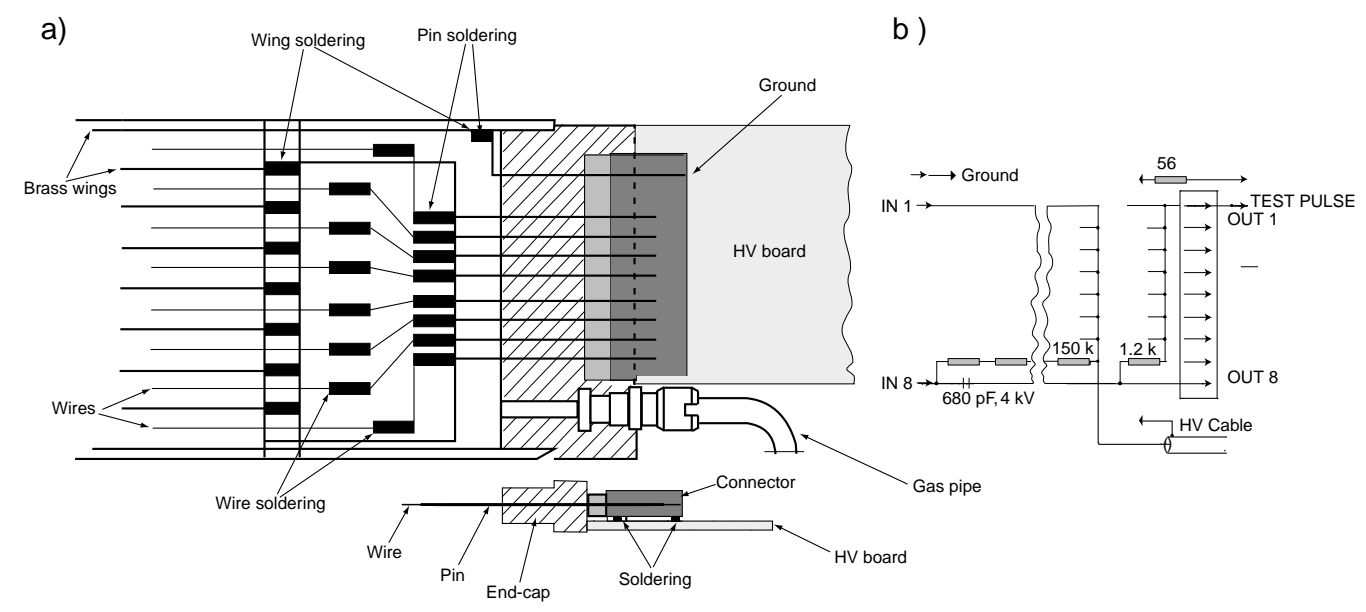

Figure 6: a) Terminal section of a chamber. It includes the printed-circuit board where the wires are soldered, the end-cap connector, and the gas pipe. b) Board for HV supply and wire signal collection.

chamber are soldered to the same printed-circuit board and fed, together with a ground connection, through a 9-pin end-cap connector. The high voltage (HV) is supplied to the wires via feed resistors mounted on a thin printed-circuit board $\left(42 \times 100 \times 6 \mathrm{~mm}^{3}\right.$, see Fig. 6) by a single HV cable directly soldered on the board. The latter is plugged into the end-cap connector. On the same board, eight decoupling HV capacitors (680 pF, one capacitor for each wire) are soldered for reading out each of the wire signals through one of the connector pins.

The chambers are operated at $3 \mathrm{kV}$ with a $55 \% / 45 \%$ by volume gas mixture of $\mathrm{CO}_{2}$ and $\mathrm{C}_{5} \mathrm{H}_{12}$ (iso-pentane at $14^{\circ} \mathrm{C}$ ). The gas supply is serial through the chambers of a layer, i.e. the output of one chamber is connected to the input of the next one by a small stainless-steel pipe. The high gain of the tubes permits them to be read out without amplification. The construction procedure for this large number of detectors is outlined in Appendix A.

\section{$2.3 \quad$ Pick-up strip plates}

The pick-up strips (two plates for each layer) collect the induced signals from the tubes as described in [7, 9]. The pick-up strips (Fig. 7a) cross the tube-wire direction at an 
angle of $\pm 30^{\circ}$ (in each layer +30 for the inner strip, -30 for the outer strip). A pick-up

a)

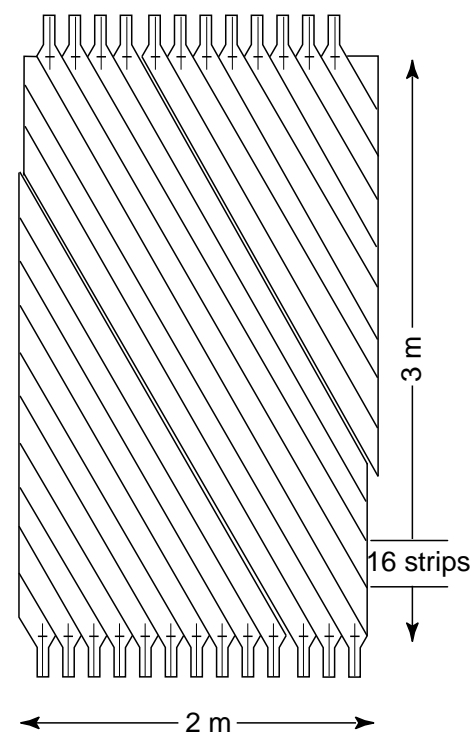

b)

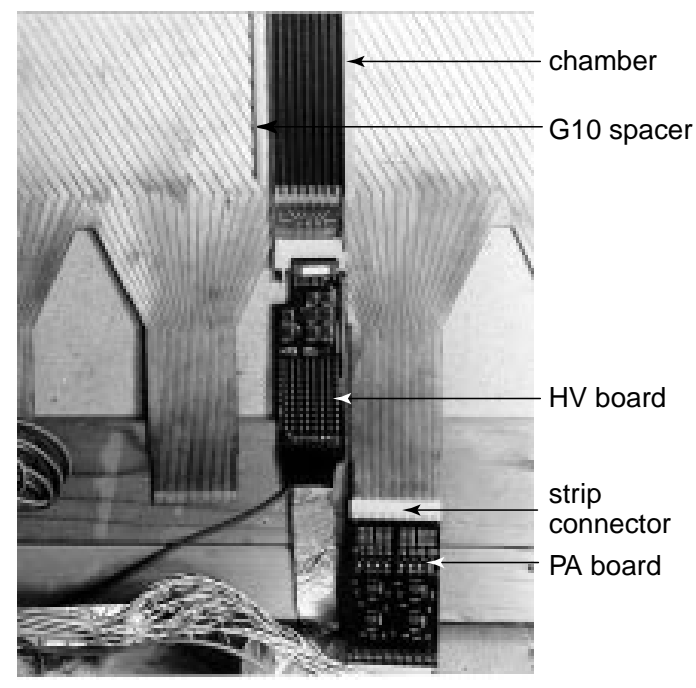

Figure 7: a) Plan view of a pick-up strip plate. The strip plates are made in 2 or 3 pieces. b) The strips are arranged in groups of 16 and reduced in size to a pitch of $2.54 \mathrm{~mm}$ to fit the connector of the read-out. Also shown is one chamber (open) with the corresponding wire read-out (HV board)

strip plate consists of a $0.6 \mathrm{~mm} \mathrm{G10} \mathrm{sheet}{ }^{7}$ coated on both sides with a $35 \mu \mathrm{m}$ thick copper layer. The copper layer facing the lead converter acts as a reference ground and shields against electric fields, while the other layer (facing the chambers) is milled in 5.6 mm wide strips at a pitch of $6.6 \mathrm{~mm}$. The area of the largest strip plates is $2 \times 3 \mathrm{~m}^{2}$, corresponding to the area of the layer with the largest radius in one azimuthal sector of $120^{\circ}$ (see Fig. 7a). The longest strips of the plates reach both ends, while the shorter ones reach only one end. Each strip is read out at only one end. On both upstream and downstream ends of the strip plates, the strips are arranged in groups of 16 and reduced in size to a pitch of $2.54 \mathrm{~mm}$ (Fig. 7b) in order to fit the connector of the read-out. The strip signals are amplified in situ by a factor of 10 with $2 \times 8$ channel preamplifier (PA) cards to approximately match the wire-signal height. This permits the use of the same digital read-out electronics as for the wires. The preamplifier cards (Fig. 8) were fitted to the strip plate by connectors, as mentioned above. The end of the strips which is not connected to the read-out is grounded via a $20 \Omega$ resistor (the strip characteristic impedance) to avoid signal reflection and to ensure good homogeneity. There is a total of 45936 strip channels.

The strips were manufactured by milling the copper with a machine developed specially for this work [10] (photographic techniques, usually used for printed-circuit boards, cannot easily be applied to such large dimensions). The machine consists of a $3 \times 1.4 \mathrm{~m}^{2}$ table equipped with a two-dimensional computer-driven arm, onto which both a milling and a cutting tool are attached. The milling precision is better than $200 \mu \mathrm{m}$, and the speed can reach several metres per minute. This enabled the required $80 \mathrm{~km}$ of milling to be

\footnotetext{
${ }^{7}$ DITRON, Novara, Italy.
} 

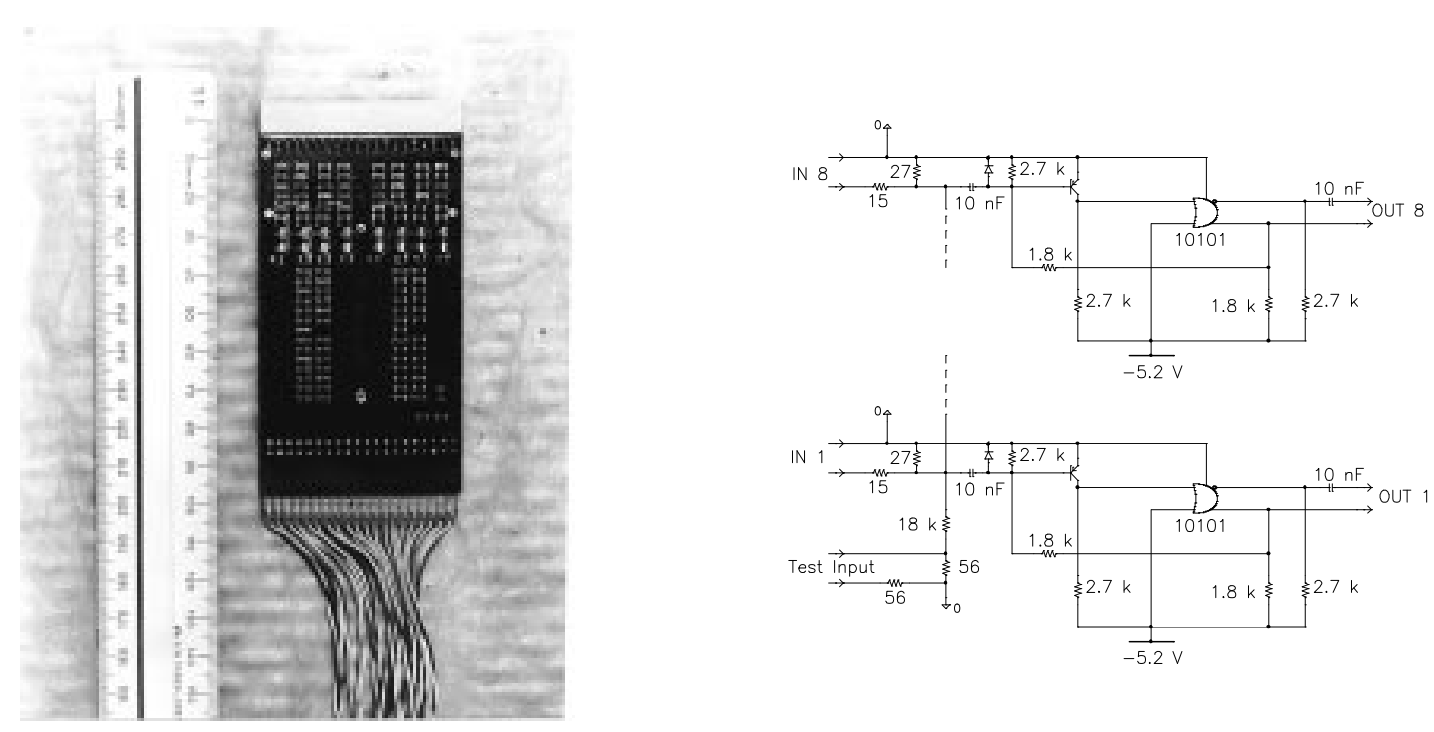

Figure 8: a) Preamplifier card for pick-up strips and b) circuit diagram.

achieved in approximately 9 months. Since the table size is smaller than that of the strip plates, the latter were made in 2 or 3 pieces (see Fig. 7a) which were later glued together along their edges.

\subsection{Strip/chamber assembly and insertion into the mechanical structure}

To maintain a good pick-up strip efficiency, it is necessary to keep a close contact between the strips and the graphited chamber covers (Fig. 9). One possibility would have been to glue the strips onto the chambers. Such a design, however, precludes the replacement of defective chambers after assembly. Instead, we preferred to insert, between sets of about 10 chambers, a $6 \times 5 \mathrm{~mm}^{2}$ G10 spacer onto which both $\mathrm{U}$ and V strips are glued but the chambers are still free to slide. These spacers introduce small dead areas in the active part of the detector. Care was taken not to align these spacers radially in the different layers; thus, the overall loss of detection efficiency is negligible.

For each sector, a calorimeter layer was assembled as follows:

- A set of chambers and the spacers were positioned on the U-strip plate. The chambers were tested for gas tightness. The spacers were glued.

- This set was put on a special cylindrical table with variable curvature. The curvature was adjusted to the expected radius of the layer. The V-strip plate was then positioned and glued to the spacers.

- The strip-chamber-strip sandwich was positioned in front of the calorimeter structure and pulled between the two corresponding converter plates. A Teflon spray was used to lower the friction between the converter plates and the copper foils of the strip plates. 


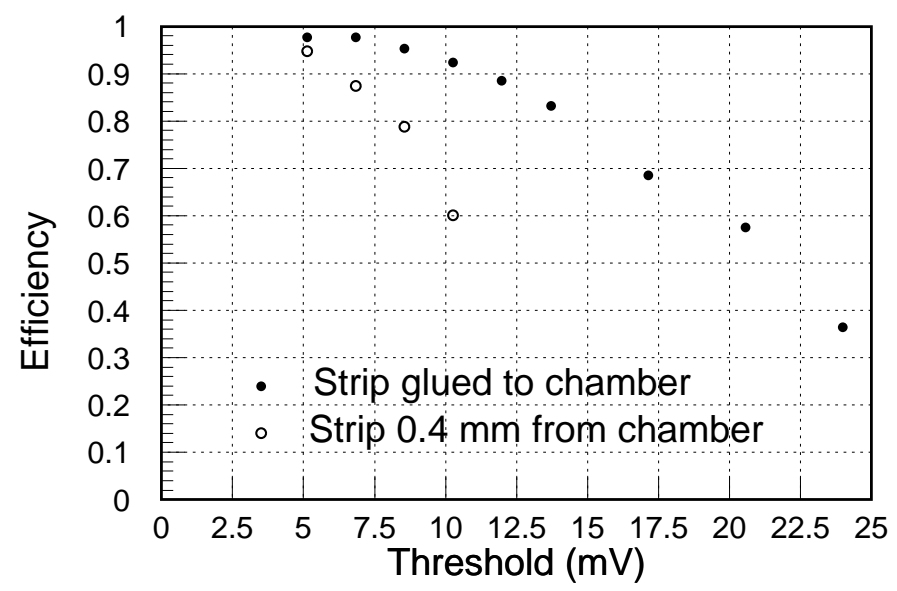

Figure 9: Efficiency of strips measured for two different spacings between the strips and the graphited chamber covers.

A schematic layout of a small part of a layer is shown in Fig. 10.

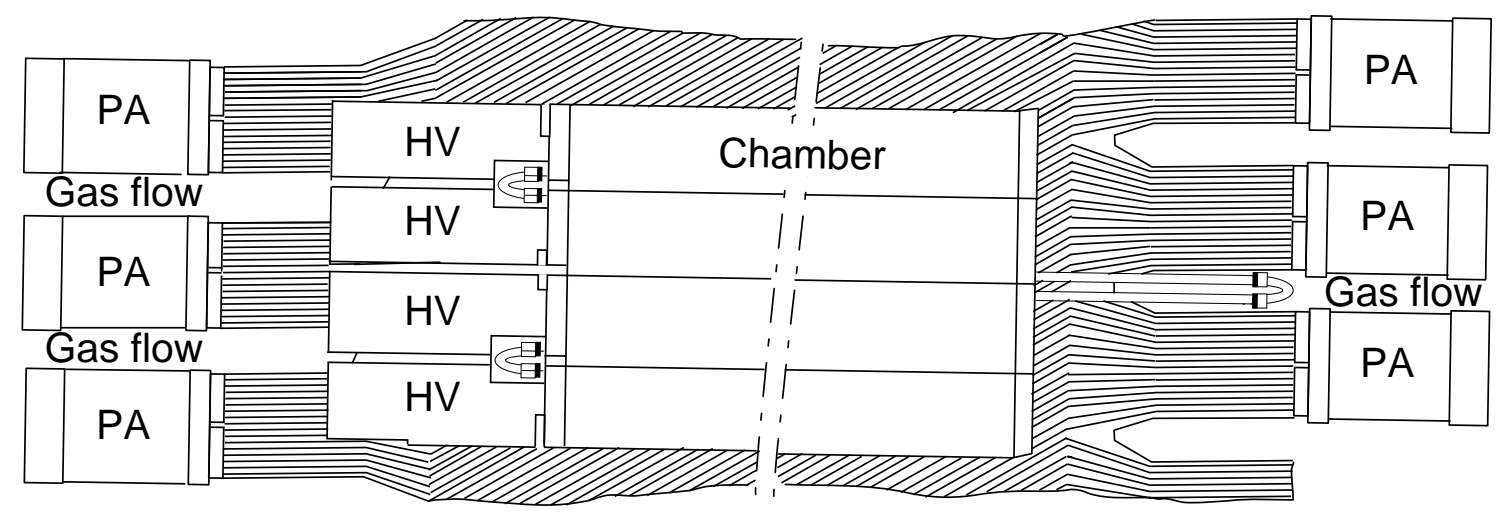

Figure 10: Arrangement of four chambers on top of the strips, showing the end parts connected to the read-out electronics as well as the pipes for gas feed-through at both ends of the chambers.

\section{Chamber Production}

After the construction of 250 chambers of $45 \mathrm{~cm}$ length for a prototype calorimeter with an active surface of $44 \times 32 \mathrm{~cm}^{2}$ and 18 layers in depth [9], 2500 chambers of $264 \mathrm{~cm}$ length were built for the final calorimeter (ECAL).

The mass production of the chambers took place at CERN and required 100 days of manual operations between February and July 1988. Several working surfaces were set up to ensure smooth and efficient production. The production was at the rate of 25 chambers per day and for 5 days per week. Each set of 25 chambers required five days 
before entering the testing procedure. The commissioning of the production unit required about two months. The technical solutions adopted for the construction as well as the operations for the assembly of these chambers, often with a certain originality, may be of interest to people undertaking similar enterprises and are therefore presented in detail in Appendix A. All the chambers (2539) which had gone through the wire-tensioning procedure reached the final stage of the test and were stored, filled with nitrogen, and ready to be used for the assembling of the calorimeter layers. Some of the chambers were kept apart for continuous testing of radiation damage on the one hand, and of the electronic interface on the other.

\subsection{Test measurements with radioactive sources, cosmic rays, and a muon beam}

The results of first tests with the produced chambers are described in detail elsewhere [7]; the main characteristics are briefly described here. The pulse shape was recorded for the wire response to electrons from a ${ }^{106} \mathrm{Ru}$ radioactive source (Fig. 2 in [7]). The pulse height was found to be typically $20 \mathrm{mV}$, and the pulse width $30 \mathrm{~ns}$. No after-pulses were observed, mainly because brass wings were used rather than more resistive ones. Spectra of the collected charge were recorded for $3.5 \mathrm{MeV}$ electrons $\left({ }^{106} \mathrm{Ru}\right)$ as well as for 5.9 $\mathrm{keV}$ X-rays $\left({ }^{55} \mathrm{Fe}\right.$ ); they look very similar (Fig. 4 in [7]), indicating that the chambers are operated in a saturated mode since the $5.9 \mathrm{keV}$ X-rays deposit about 2.5 times more energy in the gas than the electrons. Plateau curves for $\mathrm{HV}$ values in the range $2.5-3.6 \mathrm{kV}$ were recorded both with ${ }^{106} \mathrm{Ru}$ and with ambient cosmic-ray background giving similar results (Fig. 3 in [7]).

The tube efficiency was measured with a muon beam. The pulse height does not vary by more than $15 \%$ along the $2.64 \mathrm{~m}$ of the wire, except in a region of $\pm 6 \mathrm{~mm}$ around the bridges. The inefficiencies of the tubes can largely be explained by a MC simulation (see Appendix B) and are related to the geometry of the chamber, i.e. dead space due to the brass wings.

The efficiency measurements and plateau curves were repeated with a strong ${ }^{106} \mathrm{Ru}$ source delivering about $5000 \beta$ particles per second over a wire length of $5 \mathrm{~mm}$. This is about 100 times the rate expected for the experiment with the antiproton beam. The source was left at the same position for 11 days, corresponding to a total collected charge of $0.1 \mathrm{C}$. No dead-time effect was observed with this high rate and the variation of the collected charge was less than $10 \%$ over the 11-day period (Fig. 6 in [7]).

\subsection{Experience after 5 years of chamber operation}

An antiproton flux of $10^{6} \mathrm{~s}^{-1}$ was typical for the CPLEAR data-taking. The chambers were operated at $3000 \mathrm{~V}$ for the entire data-taking. The particle rate on a single chamber was on average $\approx 25 \times 10^{3} \mathrm{~s}^{-1}$ (50 tubes hit per annihilation). With a total of $2 \times 10^{13}$ antiprotons stopped in the target over a period of 5 years, the chambers were exposed to $50 \times 10^{10}$ particles. The hit distribution reaches a maximum at layers L5 to L10 and decreases gradually to about $50 \%$ of the maximum at layer L18. The rate on a strip is proportional to the length of that particular strip and thus varies by more than an order of magnitude (on average comparable to the tube rate). 
The HV monitoring system was running continuously in a mode similar to that for the conditioning of the chambers (see Appendix A), i.e. the overcurrent trip feature was active all the time. The HV of a layer with frequent trips was reduced adequately. If a chamber was faulty and could not stand an $\mathrm{HV}$ of $2800 \mathrm{~V}$, it was disconnected from the HV supply and the layer was put back to $3000 \mathrm{~V}$.

During the course of the operation, 210 chambers $(\approx 10 \%)$ had to be disconnected from the HV supply due to irrecoverable overcurrent, including $\approx 2 \%$ of the chambers which failed when the ECAL was put into operation under beam conditions. Owing to lack of physical access, the inactive chambers could not be replaced once the ECAL had been assembled and installed in the CPLEAR detector. The faulty chambers could not yet be opened for inspection, so the reason for their failure remained to be established. A break in one of the wires was considered a likely reason, based on experiences during production and commissioning which showed that a wire could break at an intermediate position (not at the soldered points). However, no firm correlation was established between a discharge from an ignition point developed on the wire or on the graphited walls. A fault in the HV card (either in the capacitor or the resistor chain) is another possible explanation.

The failure of the chambers over the five years was analysed as a function of operation time, irradiation and beam intensity during data-taking, and also in terms of geometrical aspects (layer, sector). No geometry or time dependences were observed, thus excluding mechanical stress, radiation damage, and ageing effects as possible reasons for the faults. There are some hints from the data-taking periods with high beam intensity that increasing the amount of gas flow through the chambers reduces the rate of faults.

\section{The Calorimeter Digital Read-out System}

The data acquisition system [11] of the calorimeter has to provide the information from $\approx 64000$ channels within a few $\mu$ s to the second-level trigger processor (HWP2.5 [12]), and to finish the complete read-out in $\leq 1 \mathrm{~ms}$ (see the block diagram in Fig. 11). Modern design techniques, parallel data-transfer protocols, and multi-level data reduction techniques are used to achieve the required performance.

The Fastbus (FB) standard is used throughout. The read-out is housed in standard FB crates equipped with $2 \mathrm{~kW}$ F6853 power supplies (100 kHz switching units) ${ }^{8}$, with a special shielding arrangement to minimize the noise level.

\subsection{Electronics at the detector}

As mentioned in Sections 2.3 and 2.4, the high-gain mode of the streamer tubes permits their wires to be read out without amplification, while the strip signals are amplified (gain of 10). The HV boards provide the HV to the 8 wires of each chamber, and PA boards are used to amplify the signals of the strips (16 strips per board). Both elements are constructed using surface-mount technology (SMT). On the HV boards the HV feed resistors consist of a chain of SMT resistors. The decoupling condensers $(680 \mathrm{pF})$ are of a standard HV type; however, care was taken to find the smallest size available on the market which was compatible with the $4 \mathrm{kV}$ range ${ }^{9}$. A special coating on the $\mathrm{HV}$

\footnotetext{
${ }^{8}$ Wiener, 51399 Burscheid-Hilgen, Germany.

${ }^{9}$ manufactured by Murata, Japan.
} 


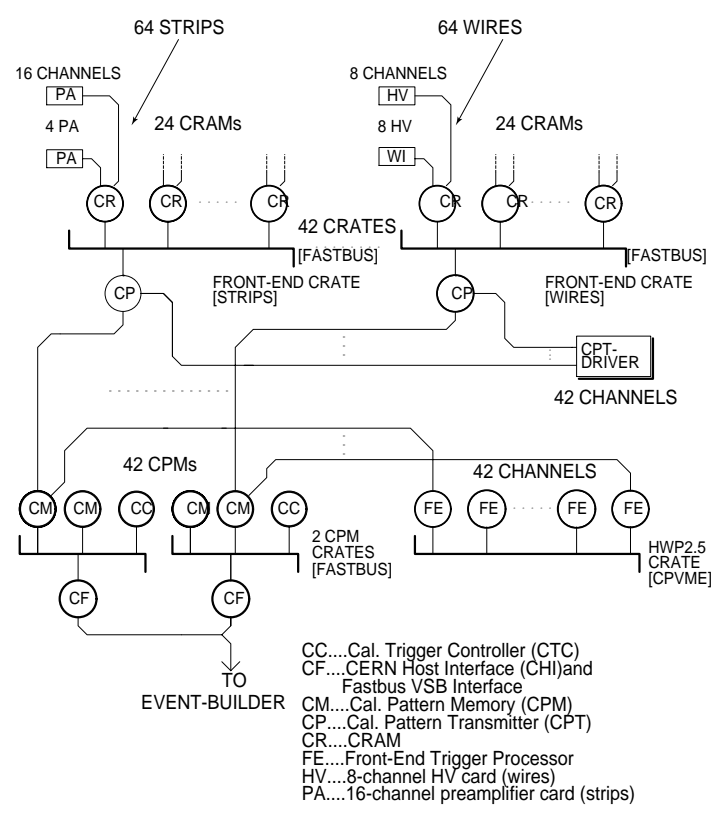

Figure 11: Block diagram of ECAL read-out.

board provides resistance to the HV. The preamplifiers use a standard ECL 10k logic gate (MC10101) in a linear mode, providing the desired gain and complementary output drivers.

The wire signals are transmitted to the front-end modules via twisted-pair cables, one side of the pair being directly driven from the wire, and both terminated via a $56 \Omega$ resistor to ground. The strip signals are transmitted in differential mode from the driver of the preamplifier. All cables (signal and HV) are routed through the gap between the magnet coil and the return yoke, and are then fixed on six annular supports which surround the magnet coils. Space constraints inside the detector made the use of flat cables (between 1 and $3.5 \mathrm{~m}$ long) necessary, with one cable per two HV boards terminated in a 37-pin connector (Cannon type). The 16-channel preamplifier board connects its twisted-pair flat cable via a mass-terminated 37-pin connector (Cannon type). The signals are then sent to the read-out via round cables of 18 twisted pairs with a drain wire as common screen (kept typically $10 \mathrm{~m}$ in length for the wires (due to the single-ended driving of the wire signals), and $16 \mathrm{~m}$ for the strips). One pair carries the test pulse from the CRAM (see next Section), and another the low voltage needed to power the strips $(-5.2 \mathrm{~V}, 10 \mathrm{~mA}$ per channel and $160 \mathrm{~mA}$ per cable). The low voltage required for the strip preamplifier is provided by external power supplies via bus bars situated at special pins on the backplane of the FB crate containing the CRAMs. Six of the low-voltage power supplies (only grounded on the detector side) are housed in a special 19-inch unit. Several of these low-voltage units are strategically placed within the 22 racks which house the calorimeter electronics. The power dissipation is $\leq 50 \mathrm{~mW}$ per channel, and no cooling is required. We were forced to use this rather inelegant system, because of the lack of space for bus bars on the detector itself.

The HV was fed by one cable per chamber, from a distribution box supplying all the chambers of one layer of a sector in parallel, but with the possibility of feeding individual 
chambers if required. We used a A433N-SY127 HV system ${ }^{10}$, equipped with 4-channel modules $(4 \mathrm{kV}, 400 \mu \mathrm{A} /$ channel, $0.2 \mu \mathrm{A}$ resolution).

\subsection{Front-end module (CRAM)}

The wire and strip signals are received by the front-end modules: Calorimeter Receiver And Memory (CRAM). Each module contains 64 channels of input receivers, discriminators, shapers, digital delays and output registers (see the block diagram in Fig. 12 and the technical description [13]). Input receivers, discriminators, and shapers for 4 channels are

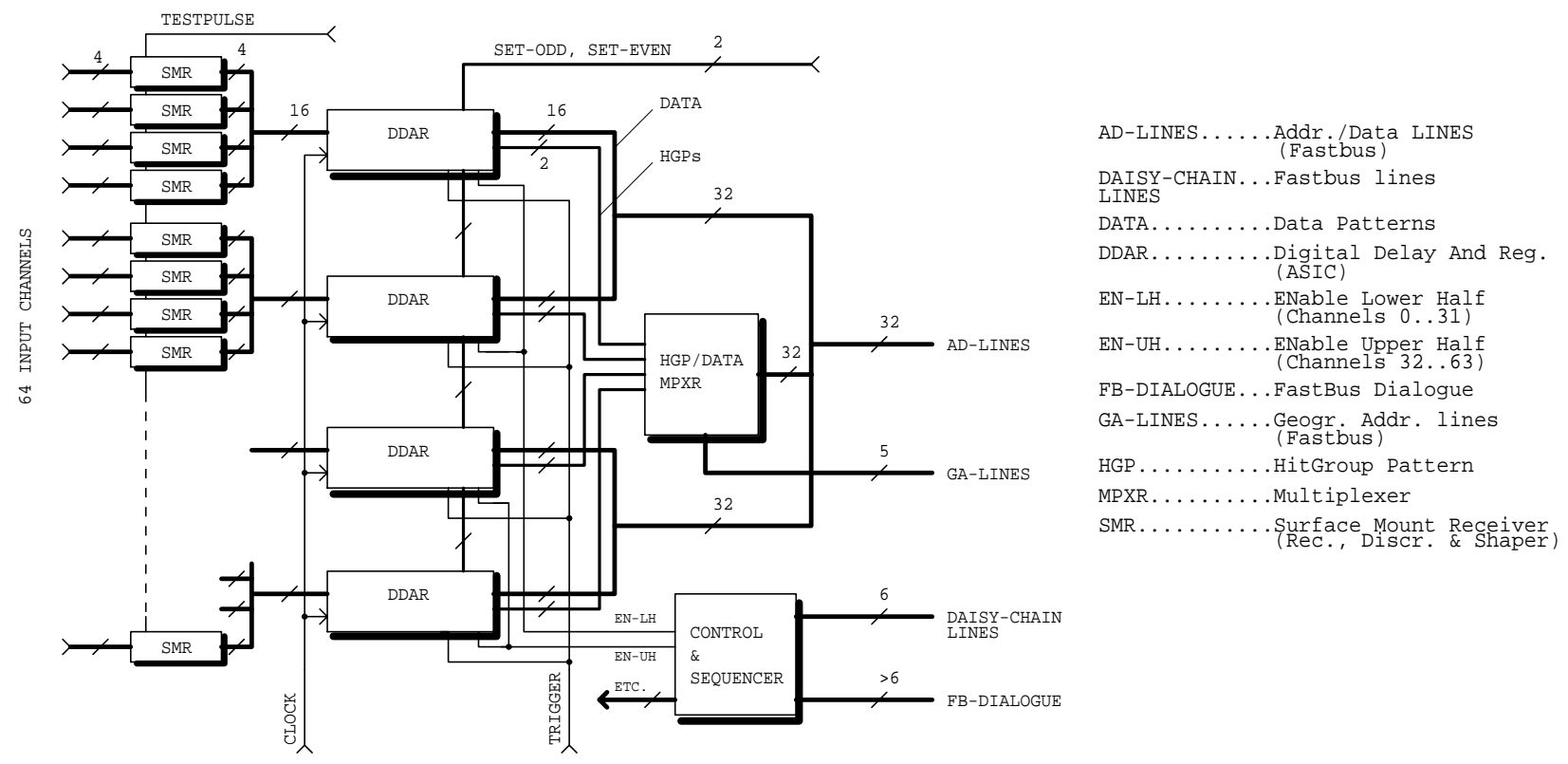

Figure 12: Block diagram of a CRAM.

contained in one SMR (surface-mount receiver) subcard designed with SMT components. They allow for easy commissioning and repair. The modules use an auto-updating mode derandomizer (every $40 \mathrm{~ns}$ ), which makes event-by-event resetting unnecessary. The delay element is a digital shift register followed by an output register. Additional features are selectable after-pulse cancellation times (set to $80 \mathrm{~ns}$ ) and selectable acceptance window widths for the output register (set to $120 \mathrm{~ns}$ ). All the above-mentioned functions have been implemented in an ASIC ${ }^{11}$ element: Digital Delay And Register (DDAR) ${ }^{12}$. Each DDAR contains 16 channels of derandomizer, 16 shift registers (20 cells deep) and 16 output register cells.

Clock and trigger signals, as well as the common discriminator threshold voltage, are provided via the special FB crate controller (CPT, see Section 4.3). The discriminator threshold can be either that provided by the CPT or can be set individually at each CRAM. Test pulses can be sent either to the input of the SMRs or to the input of the HV or PA boards. The internal output registers can be preset for odd, even or all channels. These options permit a detailed simulation of the calorimeter read-out system and enable

\footnotetext{
${ }^{10} \mathrm{CAEN}, 55049$ Viareggio, Italy.

${ }^{11}$ ASIC: Application Specific Integrated Circuit.

${ }^{12}$ designed in collaboration with Eljapex, 6300 Zug, Switzerland; manufactured by Fujitsu, Japan.
} 
its functioning and performance to be checked quickly. The low voltage for the strip preamplifiers is routed through the corresponding CRAM from the FB backplane (see Section 4.1). Each sector of ECAL is instrumented with 90 CRAMs for the wires, and 120 CRAMs for both U and V strips, which adds up to 990 CRAMs distributed in 42 crates for the three sectors, giving a total of 63360 channels.

\subsection{Special crate controller (CPT)}

The information in the CRAMs (up to 24 CRAMs are housed in one FB crate) is read out via a special crate controller - Calorimeter Pattern Transmitter (CPT) - using a daisy chain protocol. The data transfer consists of two phases: the transfer of vernier information and data pattern transfers. The vernier information (also called hitgroup bit) - the OR of 8 channels (representing exactly one chamber in the case of wires) contains the hitmap of up to 192 chambers (six 32-bit words) and is always transferred since it provides the indirect addressing information of each data pattern within each crate. Data patterns are read out in sparse data-scan mode (only non-zero patterns are transferred); they represent the exact hit distribution of 8 chambers (max. 48 32-bit words). The granularity of 8 channels was chosen to allow fast online reconstruction of showers in all three projections via the external hardwired processor HWP2.5.

A central distribution system (CPT-driver) provides signals such as clock, trigger (including corresponding delays), test pulses and common discriminator thresholds via each CPT to all CRAM modules in a crate. It is housed in a sub-chassis in a standard NIM crate.

\subsection{Processing module (CPM)}

Each of the 42 CPT modules is connected to a corresponding processing module: Calorimeter Pattern Memory (CPM). The CPM modules (see the block diagram in Fig. 13) provide for fast data collection, for sending information to HWP2.5, and for efficient encoding of data in order to minimize the amount of data which has to be written to tape. An average event of about 400 fired channels will give 100 32-bit words after encoding. The data transfer of all $42 \mathrm{CPTs}$ in parallel starts with a positive first-level trigger decision and takes 3-7 $\mu$ s depending on the number of data patterns to be transferred. The vernier information received from the CPT module can be masked with a 'hot-wire' map, thus avoiding the triggering on hot channels. It can also be used to suppress unwanted parts of the calorimeter with a granularity of 8 channels. The vernier information can be requested by the HWP2.5 via the HWP port after the masking phase. The subsequent encoding the data into 16-bit patterns takes between 3.2 and $24.3 \mu$ s depending on the number of data patterns to be treated. The encoding is performed by an ASIC array (PERAC ${ }^{13}$ ) controlled by synchronous-state machines developed in PLDs ${ }^{14}$. The chaining of the individual-state machines (processes) is controlled via asynchronous registers which are also developed in PLDs. All state machines and control circuits were simulated by using a simulator developed in-house (TPSIM [14]). The memories for the vernier information (raw, hot and masked hitgroup patterns), the data patterns (raw memory) and the output data patterns (output pattern memory) are also accessible via the FB backplane,

\footnotetext{
${ }^{13}$ PERAC: Priority Encoder Register And Counter circuit

${ }^{14}$ PLD: Programmable Logic Device
} 


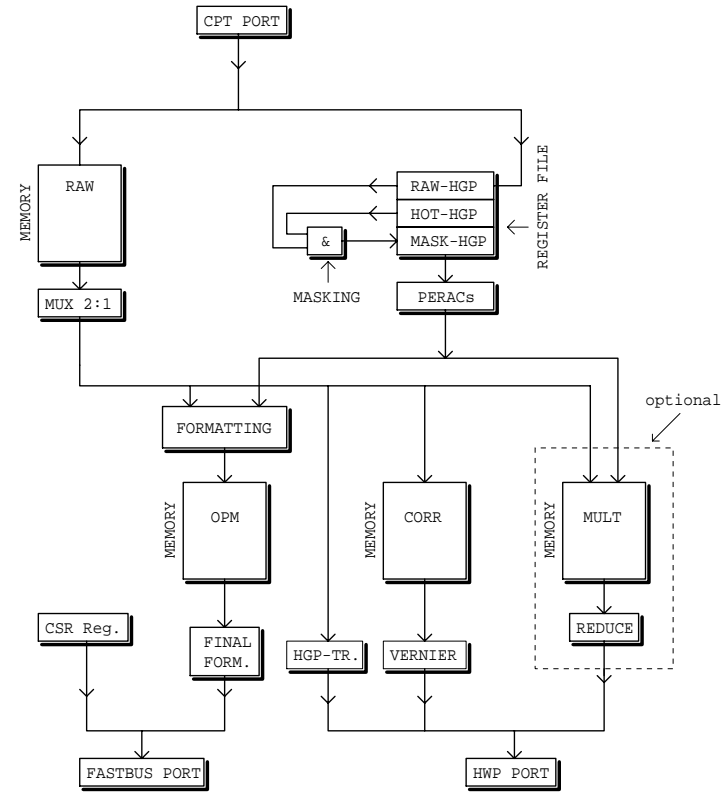

Figure 13: Block diagram of a CPM.

thus making versatile testing possible. The HWP2.5 calculates in parallel the number of showers (from the vernier information) seen by the calorimeter and decides within $17 \mu \mathrm{s}$ whether the event should be written to tape.

\subsection{Interface to the event-builder ( $\mathrm{CHI}$ )}

The final read-out is performed by two FB master modules: CERN Host Interface (CHI [15]) after the event has passed the last trigger decision. Each CHI is housed in a FB crate together with 20 and $22 \mathrm{CPM}$ modules, respectively. A special program executing on CHI was developed [16] to read out a given number of CPMs within one optimized block-transfer. Each CPM accessed will in turn perform the final data reduction (two 4-bit patterns plus address information are contained in a 32-bit data word) 'on the fly' using PERAC circuits. The identification of CPM modules within a crate is provided via a geographic address bit (FB protocol). The MSB (most significant bit) is provided via a link setting on each CPM module, which is identical in each CPM crate. The read-out of 21 modules takes $450 \mu \mathrm{s}$ for our type of events ( $\approx 100$ words $=400$ bytes $)$. The data memory of the CHI is transparent to the event-builder (in a VME crate) via a FB to VSB Interface (FVSBI) module (STR330/VSB ${ }^{15}$ ) and a receiver card (STR723 ${ }^{16}$ ) on the VME Secondary Bus (VSB) port of the event-builder. Both CHIs are connected to the same VSB port. A small fraction of the events (about 1 out of 100) are sent to an FIC 17 via Ethernet using Remote Procedure Call (RPC) to monitor the performance of the calorimeter online.

A special controller module - Calorimeter Trigger Controller (CTC) - housed in each of the two CPM crates, provides for common control signals to all CPMs, such as start data acquisition, clock, reset.

\footnotetext{
${ }^{15}$ STR330/VSB, STRUCK, 22889 Tangstedt/Hamburg, Germany.

${ }^{16}$ STR723, Differential VSB Extension, STRUCK.

${ }^{17}$ Fast Intelligent Controller, FIC 8230, CES, 1213 Petit-Lancy/GE, Switzerland.
} 
Several test modules were developed to assist the commissioning and testing of the whole acquisition chain: wire data and strip data simulator (TCP tester), CPT simulator, etc.

\subsection{Manufacturing and commissioning of the front-end electron- ics}

The HV boards (1071 pairs) and the PA boards (2871), together with their corresponding flat cables, were manufactured by an industrial firm ${ }^{18}$. The number of units was increased by $5 \%$ (spares) at the moment of the production. Members of the collaboration provided the test equipment and carried out the commissioning tests. The test included (for the preamplifiers) thermal cycling; the boards, powered with $-5.2 \mathrm{~V}$, were heated from $-10^{\circ} \mathrm{C}$ to $+70^{\circ} \mathrm{C}$ every hour, for 24 hours. The SMR subcards (16 000) and the CRAMs (1000) were manufactured by the same firm. The test equipment for SMRs and CRAMs [17] was developed at CERN. A special unit, called 'intelligent' CRAM, was developed for the testing of the CRAMs. It was equipped with a control-status register allowing the readout of the CRAMs without the need of the CPT/CPM chain. After initial tests at the manufacturer, the SMRs and the CRAMs were fully tested at CERN. A dedicated test setup including test software for the whole DAQ chain was also developed, for maintenance purposes.

\section{Performance of the ECAL}

To give an idea of what is expected to be measured in ECAL, the longitudinal and lateral shower development is displayed in Fig. 14 for MC-simulated showers originating from $150 \mathrm{MeV}$ photons which were generated to all convert in the same tube of layer L1 (Fig. 14a) or L6 (Fig. 14b). While the lateral shower development is relatively narrow, the longitudinal development extends over the entire thickness of the calorimeter, even at this low energy. Consequently, the shower losses (leaking) are fairly substantial for those photons which do not convert in the first layers.

The performance of the ECAL is characterized by the photon detection efficiency, the photon-conversion position-resolution, the energy resolution, the shower-direction resolution, and the charged-particle identification efficiency (e/ $\pi$ separation). All of these performance measurements were carried out with particles emitted from $\bar{p} p$ annihilation at rest or their decay/interaction products using one of the following data samples (only the prototype calorimeter was exposed to beams of electrons, pions, muons, and tagged photons):

- $\pi^{ \pm}$sample: the data were taken with a minimum bias trigger (coincidence required between an antiproton of the beam and one hit in one of the inner PID scintillators). The $\pi$ fraction exceeds $95 \%$ for $\bar{p} p$ annihilation at rest. This sample is directionally unbiased. The tracks penetrate all layers of ECAL. The offline selection required a clear association of the ECAL information with the hits in the tracking devices.

- $\mathrm{e}^{ \pm}$sample: $\mathrm{e}^{+}$and $\mathrm{e}^{-}$were obtained simultaneously from pair production, $\gamma \rightarrow$ $\mathrm{e}^{+} \mathrm{e}^{-}$, in the detector components [6]. Photons are abundant in minimum-bias data

\footnotetext{
${ }^{18}$ Intracom (Siber), 19400 Koropi, Greece.
} 

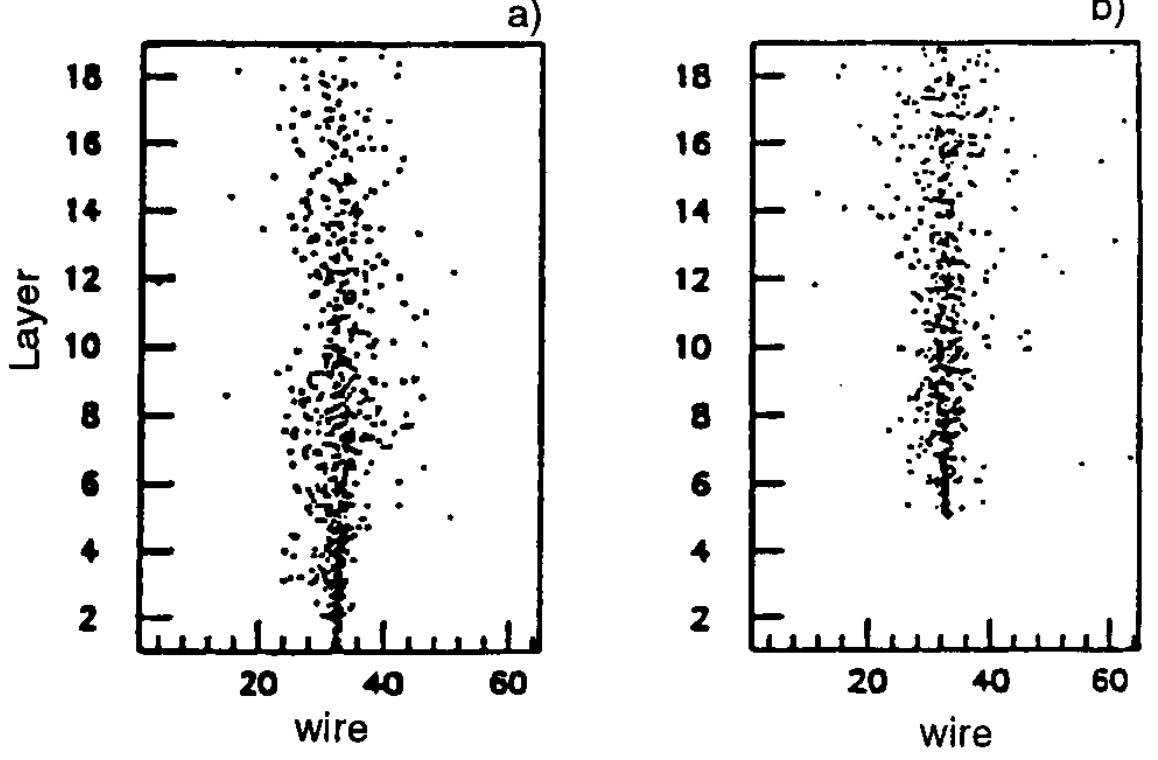

Figure 14: Scatter plot of one hundred superimposed MC-simulated showers $(150 \mathrm{MeV}$ photons), illustrating the longitudinal and lateral shower development. The simulation of all showers was started in the same tube for conversion in layer L1 a) or L6 b).

samples and have a conversion probability of about $1 \%$ in the detector region where the momentum of the $\mathrm{e}^{+} \mathrm{e}^{-}$pair can still be analysed. The signature of the $\mathrm{e}^{+} \mathrm{e}^{-}$ selection is very clean, characterized by an opening angle near to $0^{\circ}$.

- Tagged-photon sample: The two-body annihilation reaction $\overline{\mathrm{p}} \mathrm{p} \rightarrow \phi \pi^{0}\left(\phi \rightarrow \mathrm{K}^{+} \mathrm{K}^{-}\right.$ and $\left.\pi^{0} \rightarrow \gamma \gamma\right)$ as well as the annihilation process $\overline{\mathrm{p}} \mathrm{p} \rightarrow \mathrm{K}^{+} \mathrm{K}^{-} \pi^{0}\left(\pi^{0} \rightarrow \gamma \gamma\right)$ allow energy or position tagging of one of the photons by the reconstruction of the event kinematics. The $\phi \pi^{0}$ and $\mathrm{K}^{+} \mathrm{K}^{-} \pi^{0}$ candidate events are selected by requiring two charged particles in the tracking devices and at least one neutral shower in the calorimeter. The charged particles are identified to be kaons using the Cherenkov and $\mathrm{d} E / \mathrm{d} x$ information from the PID. To select the $\phi(1020)$ resonance, only events with a $\mathrm{K}^{+} \mathrm{K}^{-}$invariant mass of $1020 \pm 20 \mathrm{MeV} / \mathrm{c}^{2}$ are accepted.

As a consequence of using these data samples, some of the performance measurements are adversely affected by: 1) the momentum measurements within the CPLEAR detector, which do not have the accuracy of a dedicated beam line [8];2) particle interactions in the detector components between the annihilation region and the ECAL. Among other effects, photons are created [6] in addition to those from the $\pi^{0} \mathrm{~s}$ of neutral-kaon decay. These photons are only partly recognized and suppressed, and therefore constitute a background (typically 10\%) in some of the performance measurements.

The ECAL response did not depend on the annihilation rate (up to $10^{6} \mathrm{~s}^{-1}$ ) as long as the antiproton flux was stable. Also the presence of a magnetic field $(0.44 \mathrm{~T})$ did not change the performance. The discriminator thresholds in the CRAMs applied to the wire and strip signals were set to equivalent signal heights of 2 and $3.5 \mathrm{mV}$, respectively, unless explicitly stated differently.

At the beginning of this section, the characteristics of the wire and the strip response are presented, followed by a short description of the pattern recognition algorithm, in- 
cluding the operational definition of a neutral and a charged shower. The alignment of the chambers and the strips then completes the basic ingredients which affect the general performance of the ECAL. At the end of the section, the resolution for reconstructing the $\mathrm{K}^{0}$ decay vertex is presented using events from the $\mathrm{K}^{0} \rightarrow \pi^{0} \pi^{0} \rightarrow 4 \gamma$ decay.

\subsection{Wire and strip response}

Information on the analog signals of wires and strips was provided in Section 3.1. The efficiency and hit multiplicity, $m$, of the wires and the strips were systematically studied in all layers of the three sectors with the pion sample [18]. The wire efficiency is determined from the probability that a tube of a chamber fires if tubes of both adjacent layers fire. The strip efficiency is the probability of observing strip hits in a layer with one wire hit. The hit multiplicity for both wires and strips is the average number of hits per layer associated with one initial pion entering the ECAL. To first order, the response is found to be the same in all layers and all sectors. The numbers quoted below are averages from the $3 \times 18$ layers.

Wires: The response of the wires is characterized by high efficiency and low noise. A MC simulation with $100 \%$ wire efficiency predicts $11 \%$ hit inefficiency due to geometrical effects (dummy areas with no chambers, brass wings and plastic bridges inside the chambers). After correcting for these effects, the wire efficiency is found to be $91 \%$. No attempt was made to relate this efficiency to the number of channels dead at the time nor with the actual running conditions. Its impact on the shower efficiency will be shown in Section 5.5. The average wire hit multiplicity is 1.3. For the higher hit multiplicities $(m \geq 2)$ the spatial distribution was analysed. For $m=2$, the two hits are adjacent in $80 \%$ of the cases.

Strips: The response of the strips is different, mainly because the induced signals are much smaller before they are amplified. As a result, the strip efficiency is lower and the noise higher. The strip efficiency and noise were investigated as a function of the CRAM discriminator threshold.

The characteristics of the strip response were studied for the pion tracks in those layers where the wire hit multiplicity was $m=1$. The efficiency varies between 60 and $80 \%$, depending on the sector, and is different for $\mathrm{U}$ and $\mathrm{V}$ strips. While no $z$-dependence is found, the efficiency varies considerably as a function of the azimuthal angle $\varphi$. The effects observed in sectors T1 and T2 are fairly symmetric about the vertical detector axis and can be explained by the physical contact between the chambers and the strips (Fig. 9 ), related to the mechanical stress, which is imposed by the weight of ECAL, causing slight deformations. In sector T3, the stress on the detector components is much more uniform and therefore only little $\varphi$-dependence is found. A correlation between $U$ and $\mathrm{V}$ strip inefficiencies was not found which means that the strip inefficiency cannot be explained by low signals in the tubes. However, a little anti-correlation between $\mathrm{U}$ and $\mathrm{V}$ strip inefficiencies was observed to be understood also in terms of the distance between the chambers and the strips, as mentioned above. The probability of a strip multiplicity $m=2$ is about $10 \%$ for the $\mathrm{V}$ strips and up to $15 \%$ for the $\mathrm{U}$ strips. For $m=2$, the two hits are adjacent in $95 \%$ of the cases. The multiplicity is found to depend on the avalanche position, i.e. $m=1$ is maximal when the avalanche position is right in the middle of a strip, and $m=2$ is maximal at the edge of the strip.

The electronic noise (negligible for the wires) is significant for the strips. It is mani- 
fested as clusters of 2.1 hits average size, spread in the $\mathrm{U}$ and $\mathrm{V}$ strips of all layers and all three sectors. This adds some $15 \mathrm{kHz}$ of noise hits. In addition, large noise clusters of 20-80 hits showed up, mainly in 9 layers of sector $\mathrm{T} 1$, in $\approx 7 \%$ of the events, adding another $10 \mathrm{kHz}$ of noise hits. These hits were identified by the pattern recognition and subsequently discarded from the search for the strip shower projections.

\subsection{Pattern recognition (shower/track finding)}

Typical projections of wire hits to the $r \varphi$-plane (W projection) are shown in Fig. 15 for an electron shower and a pion track, to be identified by pattern recognition. In a first step, a
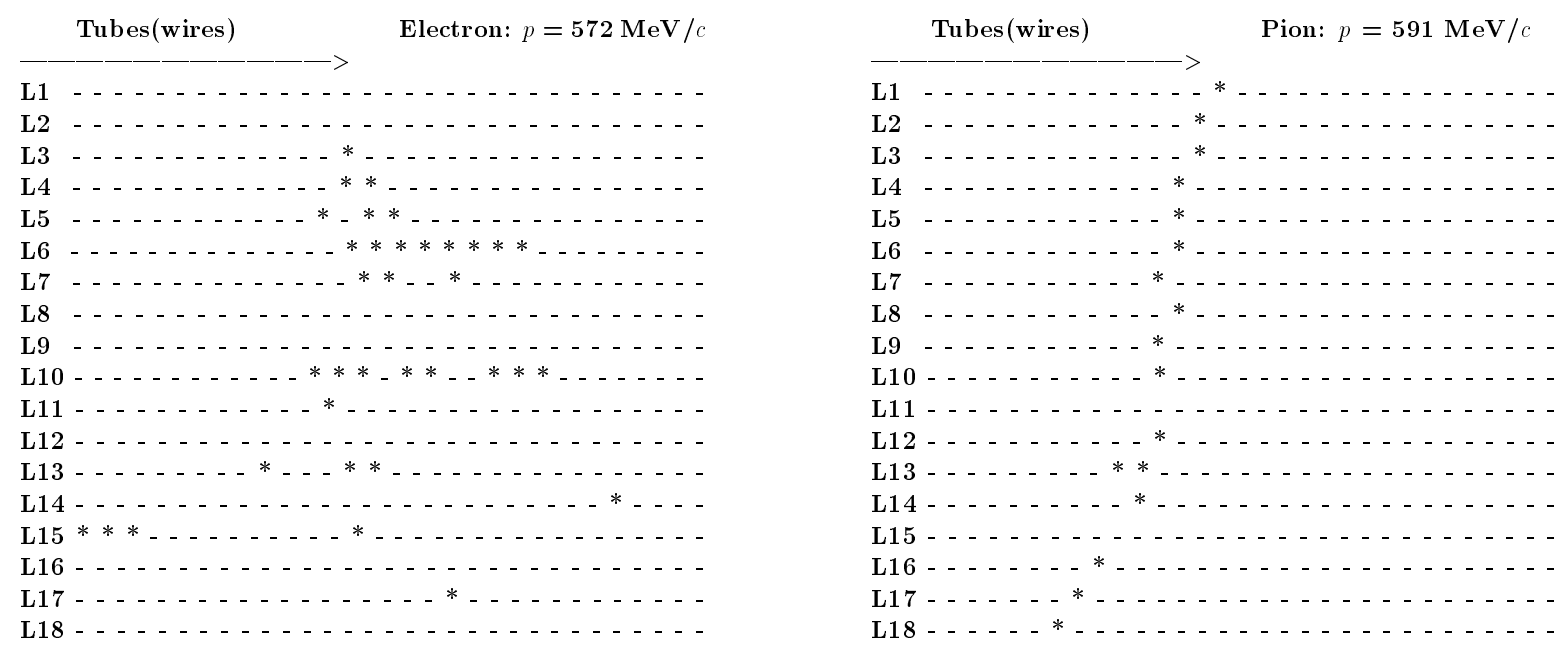

Figure 15: Shower projection of the wire hits for an electron and a pion.

tailored pattern-recognition algorithm searched for groups of hits (showers) separately in the projection of the wires (W) and the two strip planes (U, V). This search was started in layer L1 and completely ignored the track information measured for charged particles in the tracking devices. Each hit was considered either as the beginning of a new shower projection or was added to an existing one. A hit was assigned to an existing shower projection if its position was inside a position interval calculated from the hit distribution in the previous layers. The interval was defined in such a way as to also collect isolated hits and to follow the shower direction. This required a compromise between the aim of finding all hits of a shower and the need to resolve two showers nearby. There was no distinction between hits associated with charged or neutral particles traversing the ECAL, i.e. hits of charged and neutral particles were treated identically. When the search was completed at layer L18, only those shower projections were kept which fulfilled the minimal requirements $(\geq 3$ hits in $\geq 2$ layers in the $W$ projection, $\geq 2$ hits in $\geq 2$ layers in both $\mathrm{U}$ and $\mathrm{V}$ projections).

In a second step, all combinations of $\mathrm{W}, \mathrm{U}$, and $\mathrm{V}$ projections were checked for their spatial matching, taking advantage of the U,V redundancy. A shower is accepted when the spatial matching of $\mathrm{W}, \mathrm{U}$, and $\mathrm{V}$ projections is successsful $\left(\varphi_{W}-\varphi_{U V} \leq 3 \mathrm{~cm}\right)$ in one 
or several layers. The shower-foot coordinates were then obtained from the $\mathrm{W}, \mathrm{U}$, and V coordinates in the layer with the first successsful matching.

In the offline analysis, it is important to identify photon showers in the ECAL and to separate them from tracks and showers resulting from charged particles (including photon showers from secondary interactions) crossing the PID and penetrating the ECAL. To distinguish between charged and neutral showers in the ECAL, tracks are extrapolated in the magnetic field from the tracking devices through the PID into the ECAL. The shower foot of a neutral shower is separated by at least $25 \mathrm{~cm}$ in both the $r \varphi$-plane and the $z$-direction from any extrapolated track. Conversely, a charged shower is characterized by a shower foot for which the separation is less than $25 \mathrm{~cm}$.

In cases with several showers per sector, two showers may physically overlap in one of the projections $(\mathrm{W}, \mathrm{U}, \mathrm{V})$. An ambiguity flag is set if the same projection is assigned to more than one shower for the evaluation of the shower foot. This ambiguity flag is used in the offline event analysis, especially when a $\mathrm{W}$ projection is shared by two showers, since in this case the energy of the two showers cannot be determined unambiguously (see Section 5.9).

The effect of the strip noise on the pattern recognition is only minor, mainly because the wire information is so clean and because of the U,V redundancy. The shower finding was significantly affected only when the layer of the strip noise coincided with the conversion layer and the noise hits overlapped with the signal hits of the shower. The energy of a shower - determined exclusively from the wires - is not altered by the strip noise.

The different shower topology of showers associated with an electron (Fig. 15a) or a pion (Fig. 15b) will be used in Section 5.8 as a criterion for e/ $\pi$ separation.

\subsection{Alignment}

The assembly and installation of the calorimeter inside the magnet was done with a spatial accuracy of a few mm demanding offline alignment of individual chambers and strips. The alignment was performed [19] with straight-through tracks of a pion sample collected when the magnetic field was switched off (and thus without momentum information). In contrast to the standard pattern-recognition algorithm, at least five wire hits were required and only one per layer, while $\leq 3$ hits per layer were accepted for the strip projection.

The alignment of the wires was achieved iteratively, first on sets of 8 to 14 chambers, then on individual chambers, layer by layer, by comparing the $\varphi$ of the wires with the $\varphi$ of the extrapolated tracks. In a next step, the $z$-dependence of the difference $\Delta \varphi$ was evaluated and corrected for the chamber sets. The $\Delta \varphi$ distributions were fitted with Gaussians to determine the offset of the average position.

The alignment of the strips was achieved by comparing the $z$-coordinates of the extrapolated tracks with the $z$-information of U-W and V-W coordinates (obtained by crossing wires with $\mathrm{U}$ and $\mathrm{V}$ strips, respectively), layer by layer. Once again by fitting the $\Delta z$ distributions with Gaussians, the average position was determined and corrected iteratively.

Once this procedure was completed, the alignment both in $r \varphi$ and $z$ was found to be better than $0.6 \mathrm{~mm}$, which is negligible with respect to the internal resolution. The resolution of the photon conversion point measured at the Jacobian peak of the $\pi^{0} \rightarrow \gamma \gamma$ opening angle distribution with monoenergetic $\pi^{0} \mathrm{~s}$ improved by a factor of two (see Section $5.4)$. 


\subsection{Spatial resolution}

The internal $r \varphi$ resolution of the ECAL is obtained by comparing the $r \varphi$ of the wires with the $r \varphi$ information calculated with the $\mathrm{U}$ and $\mathrm{V}$ strips. The internal $z$-resolution is determined from the $z$-difference between the $\mathrm{U}-\mathrm{W}$ crossing and the $\mathrm{V}$-W crossing. The internal resolutions are $2.0 \mathrm{~mm}$ for $r \varphi$ (close to the theoretical value of $1.6 \mathrm{~mm}$ ) and $3.3 \mathrm{~mm}$ for $z$. The spatial resolutions measured with the pion sample for charged tracks and with the tagged-photon sample for electromagnetic showers are consistent with these values.

For the pion sample, tracks of at least $500 \mathrm{MeV} / \mathrm{c}$ momentum with wire/strip coordinates in the first layer (L1) of the calorimeter were selected to minimize uncertainties arising from multiple scattering. By comparing the $r \varphi$ - and $z$-coordinates of extrapolated tracks with the corresponding coordinates in the ECAL, the resolution is found to be $3.6 \mathrm{~mm}$ in the $r \varphi$-plane and $7.1 \mathrm{~mm}$ in the $z$-direction. After subtracting the contributions from multiple scattering $(2.2 \mathrm{~mm}$ for pions of $500 \mathrm{MeV} / \mathrm{c})$ and the uncertainties from the track extrapolation $(0.8 \mathrm{~mm}$ in $r \varphi$ and $6.1 \mathrm{~mm}$ in $z)$, the resolutions become $\sigma_{r \varphi}=2.7 \mathrm{~mm}$ and $\sigma_{z}=3.1 \mathrm{~mm}$.

The spatial resolution for electromagnetic showers is deduced from the photon opening angle distribution in the decay of monoenergetic $\pi^{0}$ s from the $\phi \pi^{0}$ sample. The $\pi^{0}$ total energy is $666 \mathrm{MeV}$ and the minimal opening angle is $23^{\circ}$. The measured opening-angle distribution is shown in Fig. 16 together with the MC simulation. From this simulation,

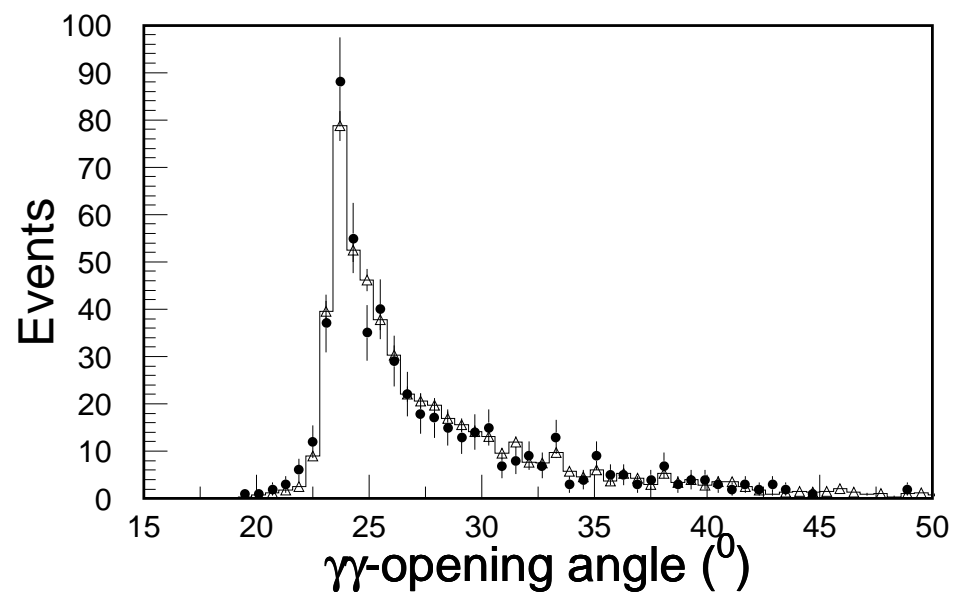

Figure 16: $\gamma \gamma$ opening angle distribution of monoenergetic $\pi^{0} \mathrm{~s}$ for real data (full dots) and MC data (histogram).

the position resolution function for $r \varphi$ and $z$ can be obtained from the difference between the true conversion coordinates and the reconstructed ones (Fig. 17). When ignoring the tails of these distributions, the $\mathrm{MC}$ resolutions in $r \varphi$ and $z$ are found to be $2.2 \mathrm{~mm}$ and $3.0 \mathrm{~mm}$, respectively. This leads to a position resolution of $\sigma_{\text {space }}^{\gamma}(\mathrm{MC})=3.7 \mathrm{~mm}$. (A simple fit of the measured distribution to the one expected from pure kinematics folded with a single Gaussian gives an opening angle resolution of $\sigma_{\text {angle }}^{\gamma \gamma}=7.9 \mathrm{mrad}$ and a 
position resolution of $\sigma_{\text {space }}^{\gamma}=4.5 \mathrm{~mm}$.)
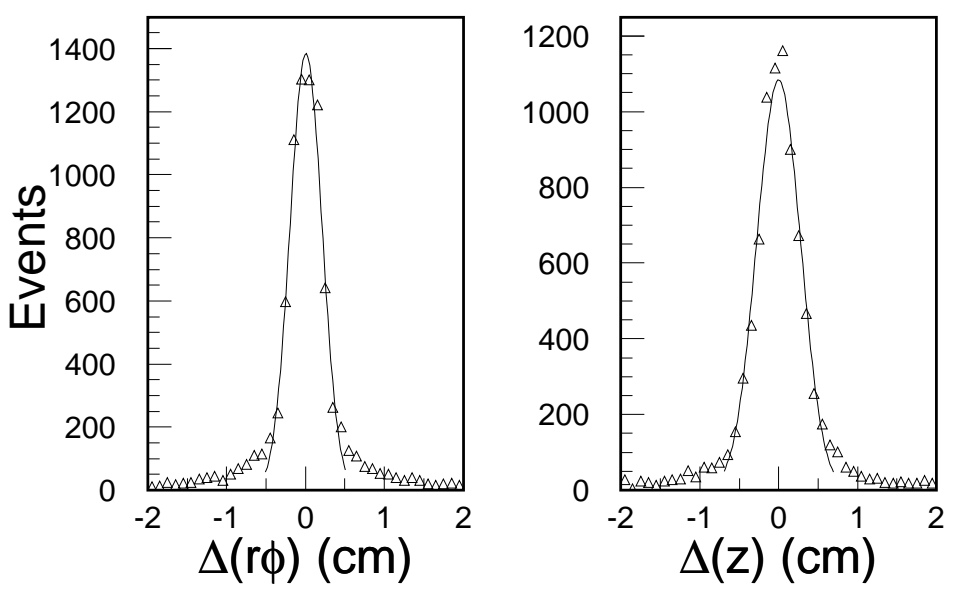

Figure 17: Position resolution obtained from MC-simulated photon showers of monoenergetic $\pi^{0}$ s: a) $\Delta(r \varphi)$, and b) $\Delta(z)$ distribution.

It should be remembered that the spatial resolution obtained in all these analyses is a convolution of the wire and strip granularity with the uncertainty of the alignment and effects related to the shower foot determination in the pattern recognition, which include the wire and strip response characteristics (efficiency, multiplicity, and noise). We expect, therefore, to obtain slightly different results for the various analyses. However, we can safely conclude, that the spatial resolution is better than $5 \mathrm{~mm}$ all but for a small fraction of showers which are in the tails of the distribution.

\subsection{Efficiency}

The shower detection efficiency is measured with tagged photons and with electrons. In both cases, real data and MC events were employed. In the analysis of tagged photons, the $\pi^{0}$ momentum is calculated from the $\mathrm{K}^{+} \mathrm{K}^{-}$momentum vector. With the position information for one detected photon, the momentum vector can be reconstructed for the other one, allowing the efficiency to be inferred by counting the events where the second neutral shower is found in the expected region. For MC-simulated events, the analysis makes use of the true photon conversion coordinates, which are then compared with the offline-reconstructed shower information. The photon detection efficiency as a function of the photon energy is shown in Fig. 18 for real data (full dots) and MC events (open triangles). Photons pointing to dummy areas (where there is either support structure or large $z$ ) were excluded from the efficiency analysis. The agreement between real data and MC events is fine for photon energies above $200 \mathrm{MeV}$, where the average efficiency is $94 \%$ for real data. The inefficiency for low-energy photons is partly due to the minimal shower requirements of the pattern recognition, but also to the detector material in front of the ECAL, which amounts to $0.05 \mathrm{X}_{0}$ for the target and the tracking devices, and 0.54 $\mathrm{X}_{0}$ for the PID. Also shown in Fig. 18 is the detection efficiency for photons converting 
exclusively inside ECAL (97\%), obtained by MC simulation and displayed by the open squares. The difference between the open squares and the open triangles accounts for shower losses due to photon conversions before ECAL.

The analysis of the MC events for which the true shower information can be compared with that from the offline-reconstructed showers allows systematic studies of the photon detection efficiency. For instance, if only layers L1 and L2 (true photon conversion) are considered, the efficiency for photon energies above $200 \mathrm{MeV}$ increases to $99 \%$. The remaining inefficiency of $1 \%$ is ascribed to the pattern recognition, partly because the inefficiency of the strips does not permit the matching of the $\mathrm{W}, \mathrm{U}$, and $\mathrm{V}$ projections. On the other hand, if events where the conversion took place before the photon reached the ECAL are included in the analysis, the efficiency drops continuously with the distance from the true conversion coordinates to the ECAL. This loss of efficiency can be explained by shower losses in the detector or reconstructed shower foot coordinates which are not found to be sufficiently close to the photon momentum direction. Furthermore, if the determination of the efficiency is constrained to events where none of the $\mathrm{W}, \mathrm{U}$, or $\mathrm{V}$ shower projections is ambiguous (ambiguity flag, see Section 5.2), the efficiency becomes only $90 \%$. The effect of ambiguity is of course dependent on the number of charged and neutral showers in the ECAL, i.e. the more showers there are in the calorimeter, the more likely it is that a projection will be used twice for the matching.

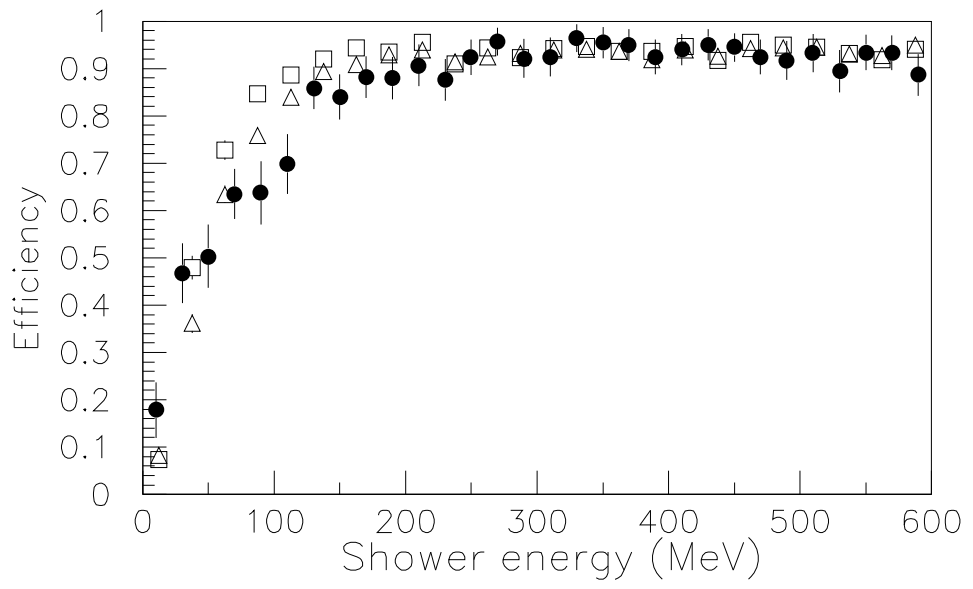

Figure 18: Photon detection efficiency as a function of $E_{\gamma}$ for real data (full dots) and MC-simulated events (open triangles), including shower losses due to photon conversions before ECAL (mainly in the PID, see text). The open squares display the MC-simulated detection efficiency for conversions exclusively inside ECAL.

The detection efficiency of the ECAL for electrons and positrons is measured with the $\mathrm{e}^{ \pm}$data sample. The tracks, measured by the drift chambers, are extrapolated through the PID, where substantial energy is deposited (ionization losses for minimum ionizing particles amount to $40 \mathrm{MeV}$ ). As a result, the detection efficiency is low for electron/positron energies below $150 \mathrm{MeV}$ (Fig. 19). The detection efficiency is the same for electrons and positrons and reaches its plateau above $200 \mathrm{MeV}$. The measured efficiency $(\geq 95 \%$ 
for energies above $200 \mathrm{MeV}$ ) is in good agreement with the MC simulation ( $\geq 98 \%$ for energies above $200 \mathrm{MeV}$ ).

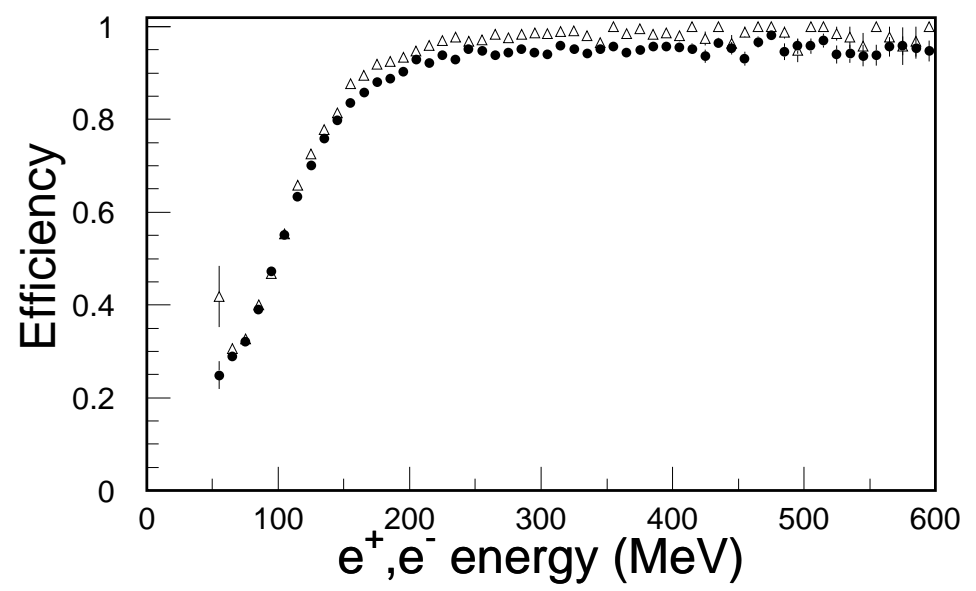

Figure 19: Electron/positron detection efficiency as a function of $E_{e}$ for real data (full dots) and MC-simulated events (open triangles).

\subsection{Energy calibration and resolution}

The energy resolution is studied [20] by means of tagged photons from the $\mathrm{K}^{+} \mathrm{K}^{-} \pi^{0}$ $\left(\pi^{0} \rightarrow \gamma \gamma\right)$ data sample. Exactly two neutral showers are required in the calorimeter. The events are passed through a 3C-fit with kinematical and geometrical constraints from energy and momentum conservation, the conversion points of the two showers, and assuming a missing $\pi^{0}$ mass for the neutral particle produced in the annihilation together with $\mathrm{K}^{+} \mathrm{K}^{-}$. As a result of this fit, the energy for the two neutral showers is obtained with a resolution better than $4 \%$, determined from MC data. The energy information from the fit is then used to calibrate the response of the calorimeter, i.e. the number of wires showing a signal (wire hits) as a function of the photon energy. The calibration must be done for all layers in which photons have converted since the shower leakage increases gradually for showers situated deeper inside the calorimeter (see Fig. 14).

Energy calibration: The measured hit distributions, i.e. the frequencies for observing a certain number of wire hits, $w$, caused by showers from photons of a definite energy $E$, deviate remarkably from symmetric curves, as seen from Fig. 20. This is partly due to the requirement that a shower has to show at least three wires with a signal.

To parametrize these hit distributions, a dedicated funtion $H(w, E)$ had to be constructed:

$$
H(w, E)=N(E) \exp \left(\frac{-\left[w-w_{0}(E)\right]^{2}}{2 \sigma_{0}^{2}(E)+(1 / 2) \Omega(E)\left[\left(w-w_{0}\right)+\left|w-w_{0}\right|\right]}\right) .
$$

$H(w, E)$ is Gaussian on one side of its maximum and becomes asymptotically exponential on the other side. The parameters $w_{0}, \sigma_{0}$, and $\Omega$ control the position of the maximum 

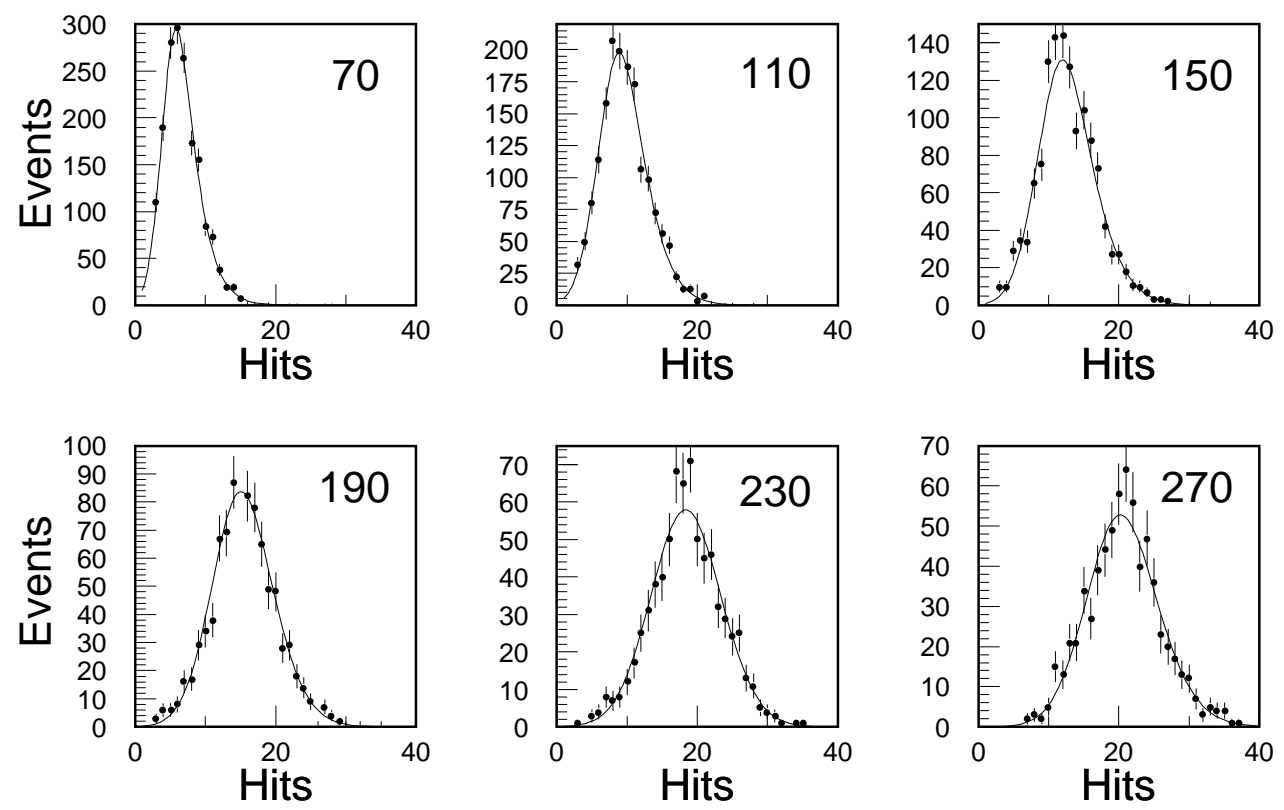

Figure 20: Hit distributions for tagged photons, converting in the first layer (L1) of the calorimeter, with energies ranging from $70 \mathrm{MeV}$ up to $270 \mathrm{MeV}$. The curves represent $H(w, E)$ (see text).

and the half-widths on the left- and right-handside, respectively. $N(E)$ is a normalization factor which serves to make $\int d w H(w, E)=1$. For $\Omega=0, H(w, E)$ is Gaussian. The parameters are evaluated from the calibration data as a function of the photon energy $E$.

For photon energies $E>250 \mathrm{MeV}$, the measured hit distributions become Gaussian with a variance of $\sigma_{0}^{2}(E)$. The mean value of the hit distribution, $\langle w(E)\rangle$, is parametrized as a function of the photon energy $E$, by the coefficients $a, b, c$ :

$$
\langle w(E)\rangle=\int w \cdot H(w, E) \cdot d w=a+b E+c E^{2} .
$$

The hit distributions $H(w, E)$ were fitted in the energy range 70 to $430 \mathrm{MeV}$ in intervals of $\pm 10 \mathrm{MeV}$ to determine the parameters $\sigma_{0}(E), \Omega(E), w_{0}(E)$, and $\langle w(E)\rangle$. Figure 21 displays the results obtained from data with photon conversion in layer L1. In turn, the polynomial fit through the data points yields the energy dependence of the parameters, e.g. $a, b, c$ for $\langle w(E)\rangle$. This fitting procedure is repeated for the other conversion layers up to layer L11 (the fit was no longer possible for higher layers because of limitations from statistics).

In Fig. 22, the variation of the mean value $\langle w(E)\rangle$ is displayed as a function of the photon energy for the conversion layers L1, L4, and L8. The observed deviation from a linear relation between the photon energy and $\langle w(E)\rangle$ or $w_{0}(E)$ reflects the moderate thickness of the ECAL (only $6 \mathrm{X}_{0}$ ), which is associated with shower leakages, especially for photons converting in layers further out in the radial direction. The fit parameters are then fitted as a function of the conversion layer. Because of the smooth behaviour of 

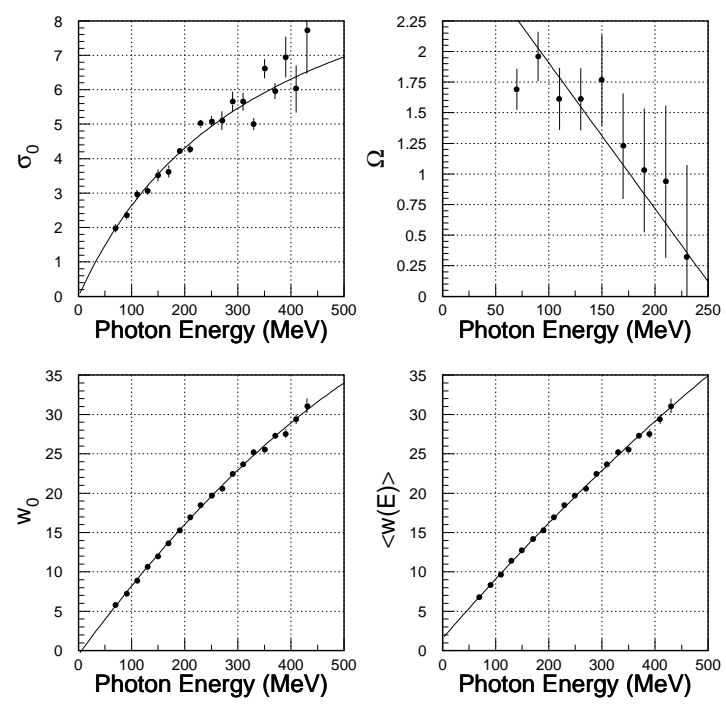

Figure 21: Energy dependence of the fit parameters defining the hit distribution $H(w, E)$, evaluated for photon conversion in layer L1. The curves are polynomials.

the calibration parameters, corresponding values for the higher layers can be inferred by extrapolation.

The energy calibration was also performed for photons converting in the PID in front of the ECAL. Separate data samples with photons converting in S1, C, or S2 (see Fig. 23) were analysed as for photons converting in the ECAL layers. The calibration for photons converting in $\mathrm{S} 2$ is found to be not much different from that for photons converting in layer L1. This is so because the amount of material (in terms of radiation lengths) between S2 and layer L1 is fairly small and not much shower energy is lost. Progressively more energy is lost for photons converting in $\mathrm{C}$ and in $\mathrm{S} 1$, because electrons of the shower lose considerable energy in the liquid of the Cherenkov. As a result, the mean of the hit distribution $\langle w(E)\rangle$ is lower for these cases than for conversion in layer L1 or in S2.

In the physics analysis, one is confronted with the opposite situation, namely to determine the energy distribution for a given number of detected hits. This is possible when the source of the photons is known, for instance $\pi^{0} \rightarrow \gamma \gamma$. In general, however, the energy distribution cannot be determined for a given number of hits. For practical reasons, therefore, the energy of a detected photon is determined by assuming that the number of hits, $w$, is equal to the mean value of the hit distribution. With this assumption, the number of hits is easily converted to a photon energy $E_{\gamma}$. In addition, the parameters describing the hit distribution $H\left(w, E_{\gamma}\right)$ for the corresponding mean value $w_{0}$ are calculated. This allows an effective variance $\sigma_{w}^{2}=\sigma_{0}^{2}(E)+2 \Omega^{2}(E)$ to be obtained, and the error $\Delta E_{\gamma}$ of the photon energy $E_{\gamma}$ to be calculated.

Energy resolution: The effective variance, $\sigma_{w}^{2}$, is found to be a good approximation for the resolution of the asymmetric hit distributions at lower energies $\left(E_{\gamma}<250 \mathrm{MeV}\right)$. The reduced resolution $R \equiv \sigma_{w} \sqrt{E(\mathrm{GeV})} /\langle w(E)\rangle$ is displayed in Fig. 24 for layers L1, L4, and L8. It is almost constant over a wide energy range - approximated by $R \approx 13 \%$ - and is found to depend only weakly on the conversion layer (up to layer L10).

Checking the energy calibration: The analysis of the $\mathrm{K}^{0} \rightarrow \pi^{0} \pi^{0} \rightarrow 4 \gamma$ decays requires 


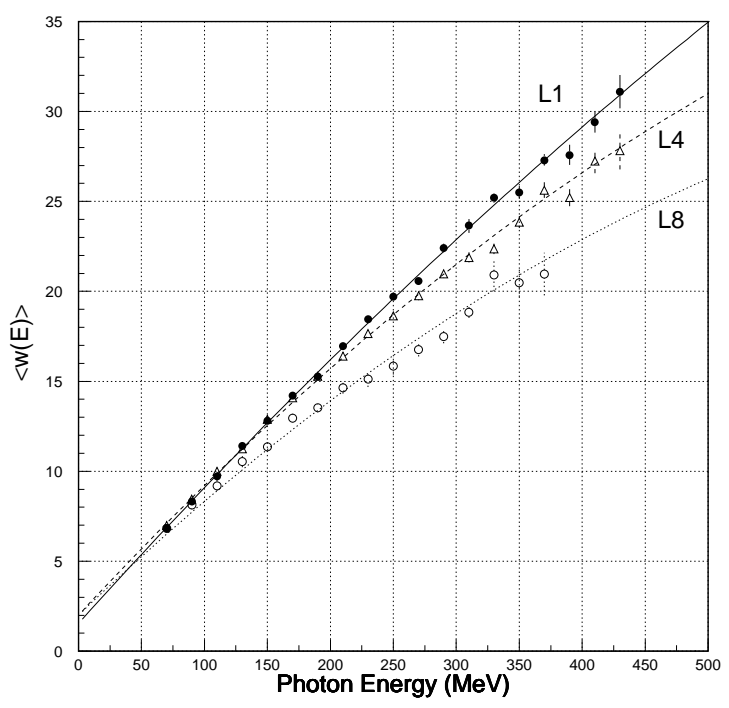

Figure 22: Variation of the mean value $\langle w(E)\rangle$ displayed as a function of the photon energy for the conversion layers L1, L4, and L8. The curves are polynomials.
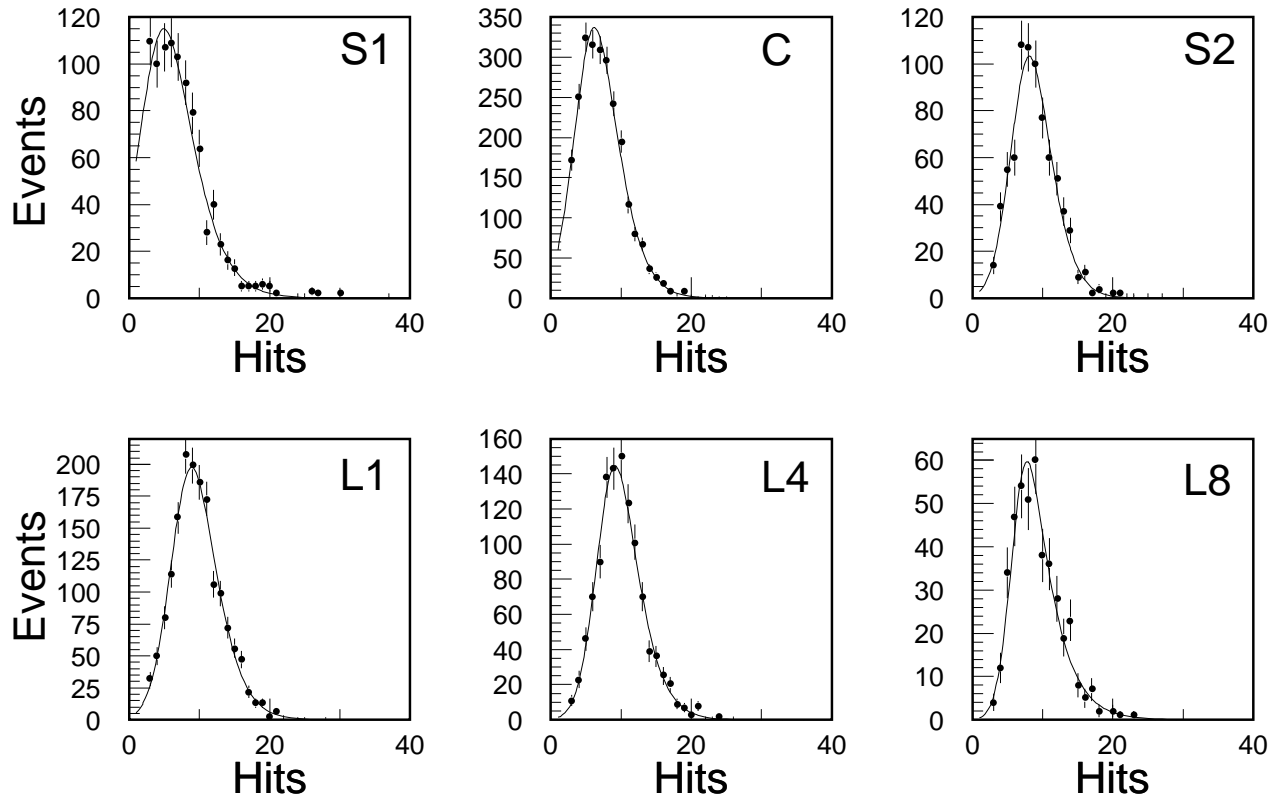

Figure 23: Hit distributions for tagged photons of $110 \pm 10 \mathrm{MeV}$ converting in S1, C, and S2 of the PID (top row), and in the ECAL layers L1, L4, and L8 (bottom row). The curves represent $H(w, E)$. 


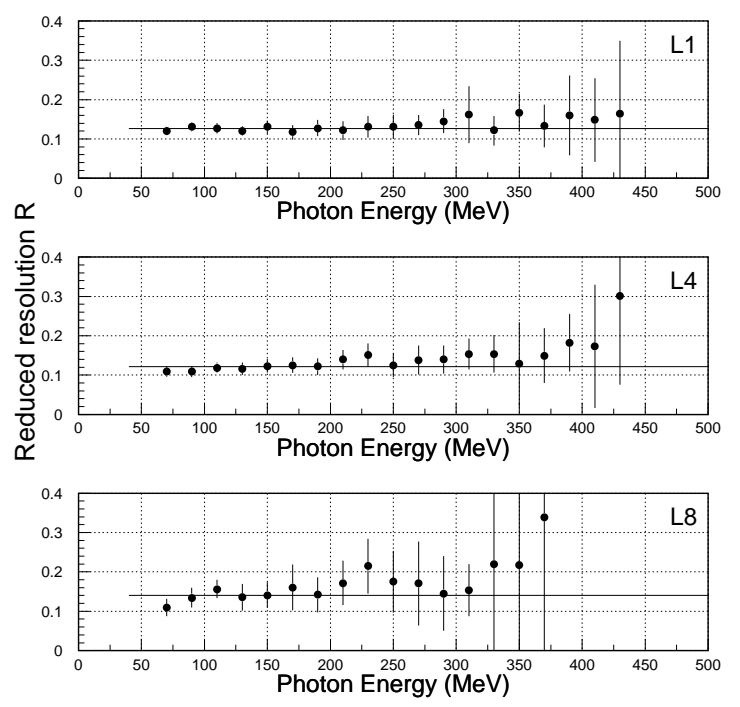

Figure 24: Reduced energy resolution as a function of the photon energy for the photon conversion layers L1, L4, and L8.

knowledge of the energy calibration in a region up to $700 \mathrm{MeV}$ where no photons from the calibration sample are available $(\geq 350-450 \mathrm{MeV}$, depending on the conversion layer). Instead, the fit of the calibration parameters is extrapolated to higher energies and the result of the extrapolation is used in these cases. The validity of this procedure was verified with photons from $\mathrm{K}^{0} \rightarrow \pi^{0} \pi^{0} \rightarrow 4 \gamma$ decays (real data and MC events). In analogy to the $\mathrm{K}^{+} \mathrm{K}^{-} \pi^{0}$ calibration data, hit distributions in intervals of $\pm 10 \mathrm{MeV}$ were generated in the energy range 60 to $700 \mathrm{MeV}$ according to the energy information from a constraint fit analysis (see Section 5.9). The result of this analysis is displayed in Fig. 25 in fine agreement with the calibration results. (Note that the energy calibration is used in the constraint fit. Therefore, the $\mathrm{K}^{0} \rightarrow \pi^{0} \pi^{0} \rightarrow 4 \gamma$ events do not qualify as calibration data. The energy constraint is however weak enough to still allow a check.)

The $\mathrm{K}^{0} \rightarrow \pi^{0} \pi^{0} \rightarrow 4 \gamma \mathrm{MC}$ events are analyzed in two ways, namely according to the energy information from the constraint fit analysis (same as for real data) and from the true input values. The hit multiplicity for MC events is found to be $10 \%$ lower than for real photon events, irrespective of the photon energy, and an appropriate correction was applied. Once this correction is done, the MC energy information on the parameter $\langle w(E)\rangle$ is in very good agreement with that found in the calibration data (see Fig. 25). The overall agreement supports the approach of extrapolation for the energy calibration.

\subsection{Shower direction}

For any charged or neutral shower, the hit distributions of $\mathrm{W}$, $\mathrm{U}$, and $\mathrm{V}$ projections allow the shower direction to be determined [21, 22] and thus provide useful information in addition to the shower energy and the conversion point. The resolution of the shower direction is expected to be a function of the total number of $\mathrm{W}$, $\mathrm{U}$, and $\mathrm{V}$ hits defining the shower. It is, of course, very poor for showers just fulfilling the minimal requirements 


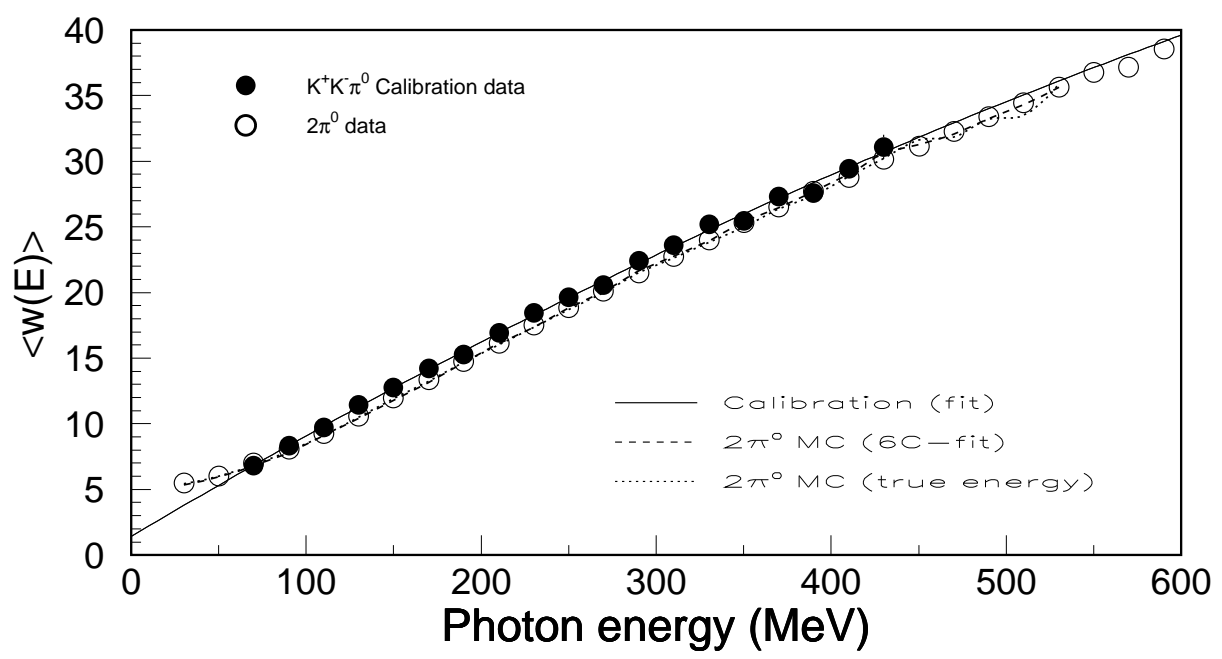

Figure 25: Energy dependence of the parameter $\langle w(E)\rangle$ displayed for $\mathrm{K}^{+} \mathrm{K}^{-} \pi^{0}$ calibration data, as well as for $\mathrm{K}^{0} \rightarrow \pi^{0} \pi^{0} \rightarrow 4 \gamma$ events from real data and MC-generated events.

of the pattern recognition, but improves with increasing shower length.

The direction of a shower is expressed by the angles $\phi^{\prime}$ and $\theta^{\prime}$ in a local coordinate system $\left(x^{\prime}, y^{\prime}, z^{\prime}\right)$ of which the shower foot represents the origin. The shower angles $\phi^{\prime}$ and $\theta^{\prime}$ are measured in the planes transverse $\left(x^{\prime}-y^{\prime}\right)$ and parallel $\left(x^{\prime}-z^{\prime}\right)$ to the beam, respectively. The shower coordinates for each layer are defined as the average hit position. While $\phi^{\prime}$ is determined by simply fitting a straight line through the W coordinates as a function of the layer, the angle $\theta^{\prime}$ follows from a straight-line fit through shower coordinates calculated from combined $\mathrm{W}, \mathrm{U}$, and $\mathrm{V}$ information.

The resolution of the shower angles $\phi^{\prime}$ and $\theta^{\prime}$ was studied with neutral showers of well known direction. The same sample of photons was used as for the energy calibration. Figure 26 shows the results for three different photon energies. As expected the resolutions improve for higher energies. Results of the reduced resolutions $\sigma_{\phi^{\prime}} \sqrt{E(\mathrm{GeV})}$ and $\sigma_{\theta^{\prime}} \sqrt{E(\mathrm{GeV})}$, where $\sigma_{\phi^{\prime}}$ and $\sigma_{\theta^{\prime}}$ denote the rms values of the resolution distributions, are displayed in Fig. 27.

The shower conversion point obtained by fitting $\phi^{\prime}$ and $\theta^{\prime}$ is in general not identical with the shower foot determination described in Section 5.2 and is found to be less accurate.

In the analysis of $\mathrm{K}^{0} \rightarrow \pi^{0} \pi^{0} \rightarrow 4 \gamma$ events (Section 5.9), the measured shower angles $\phi^{\prime}$ and $\theta^{\prime}$ as well as their covariances $\sigma_{\phi^{\prime}}$ and $\sigma_{\theta^{\prime}}$ are used. It is found that the covariances depend linearly on the angle errors returned from the straight-line fits. In addition, a constant term, which we interpret as wide-angle Coulomb scattering of electrons or positrons at an early stage of the shower evolution, has to be added. The parameters of the constant and linear terms are calibrated for photons with different number of $\mathrm{W}$ hits and conversion layers. The constant terms are generally decreasing with higher number of $\mathrm{W}$ hits and with higher conversion layer and are varying between 0.15 and $0.05 \mathrm{rad}$ for $\sigma_{\phi^{\prime}}$ and between 0.3 and $0.05 \mathrm{rad}$ for $\sigma_{\theta^{\prime}}$, respectively. In contrast, the linear terms are rising with higher number of $\mathrm{W}$ hits and do not depend on the conversion layer. The 

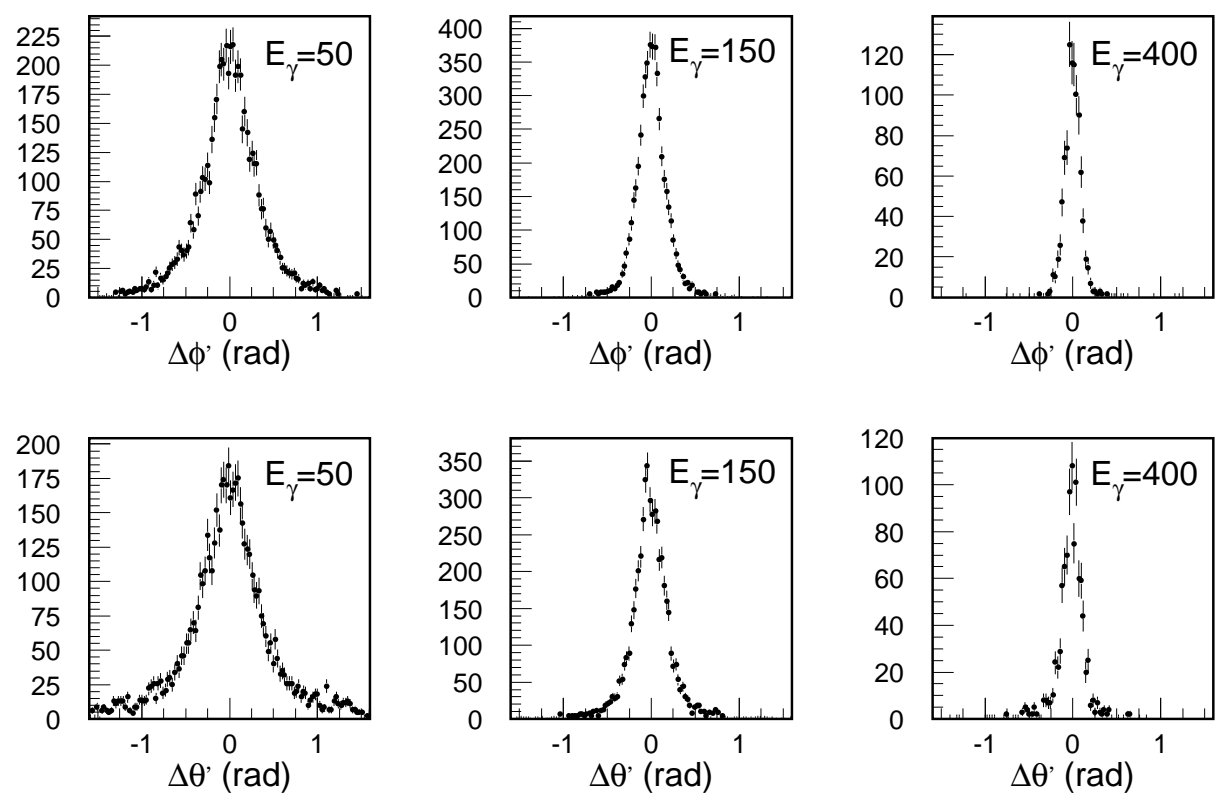

Figure 26: Distributions of the shower-angle resolutions $\Delta \phi^{\prime}=\phi_{\text {measured }}^{\prime}-\phi_{\text {true }}^{\prime}$ (top) and $\Delta \theta^{\prime}=\theta_{\text {measured }}^{\prime}-\theta_{\text {true }}^{\prime}$ (bottom) for three different photon energies $(50 \pm 25,150 \pm 25$ and $400 \pm 25 \mathrm{MeV})$.

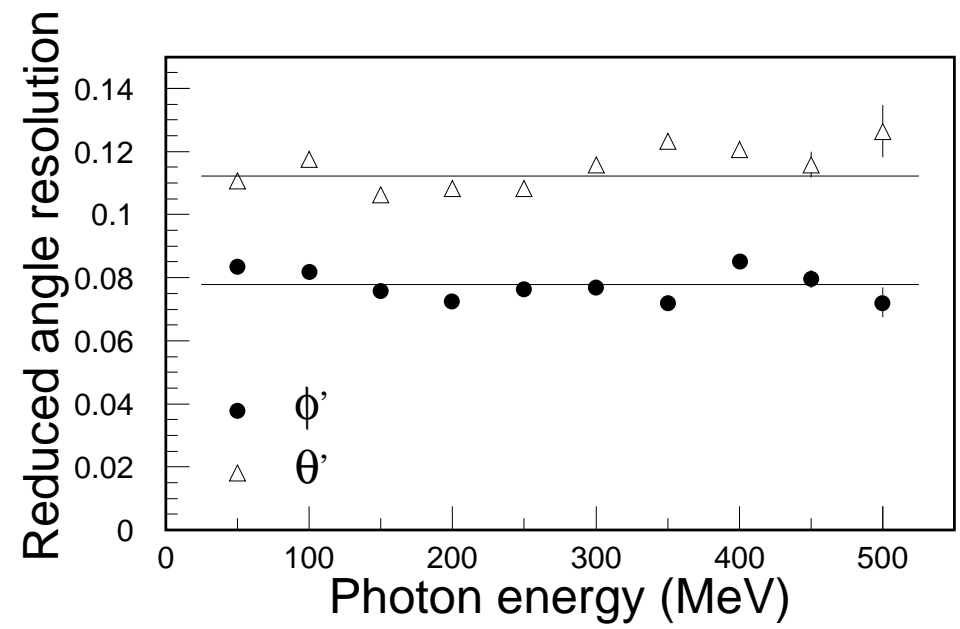

Figure 27: Reduced resolution of the shower angles $\phi^{\prime}$ and $\theta^{\prime}$ as a function of the photon energy. 
measurement errors of $\phi^{\prime}$ and $\theta^{\prime}$ are found to be uncorrelated, so that the covariance matrix is diagonal.

\section{$5.8 \mathrm{e} / \pi$ separation}

The showers associated with pions or electrons can be distinguished by their topology (Fig. 15). The neural network technique [23] was applied for the separation, using the ECAL pattern information as input and relying exclusively on the wire hits because of their low noise and high efficiency. Calibration data from $\bar{p} p$ annihilation to four or six charged pions, as well as from converted photons $\left(\gamma \rightarrow \mathrm{e}^{+} \mathrm{e}^{-}\right)$were used for training the network. The purity of these samples was better than $0.3 \%$. Relevant information for characterizing the event topology was obtained from the longitudinal/lateral shower development, i.e. the shower depth, the hit multiplicity per layer, the cluster size, etc. The training was performed in the momentum range 200 to $600 \mathrm{MeV} / c$ with $50 \mathrm{MeV} / c$ bins. At lower momenta $(\leq 200 \mathrm{MeV} / c)$, pions do not reach the ECAL. With the calibration data, an efficiency of 70 to $80 \%$ for identifying electrons was achieved over the entire momentum range (Fig. 28), at $4 \%$ pion contamination.
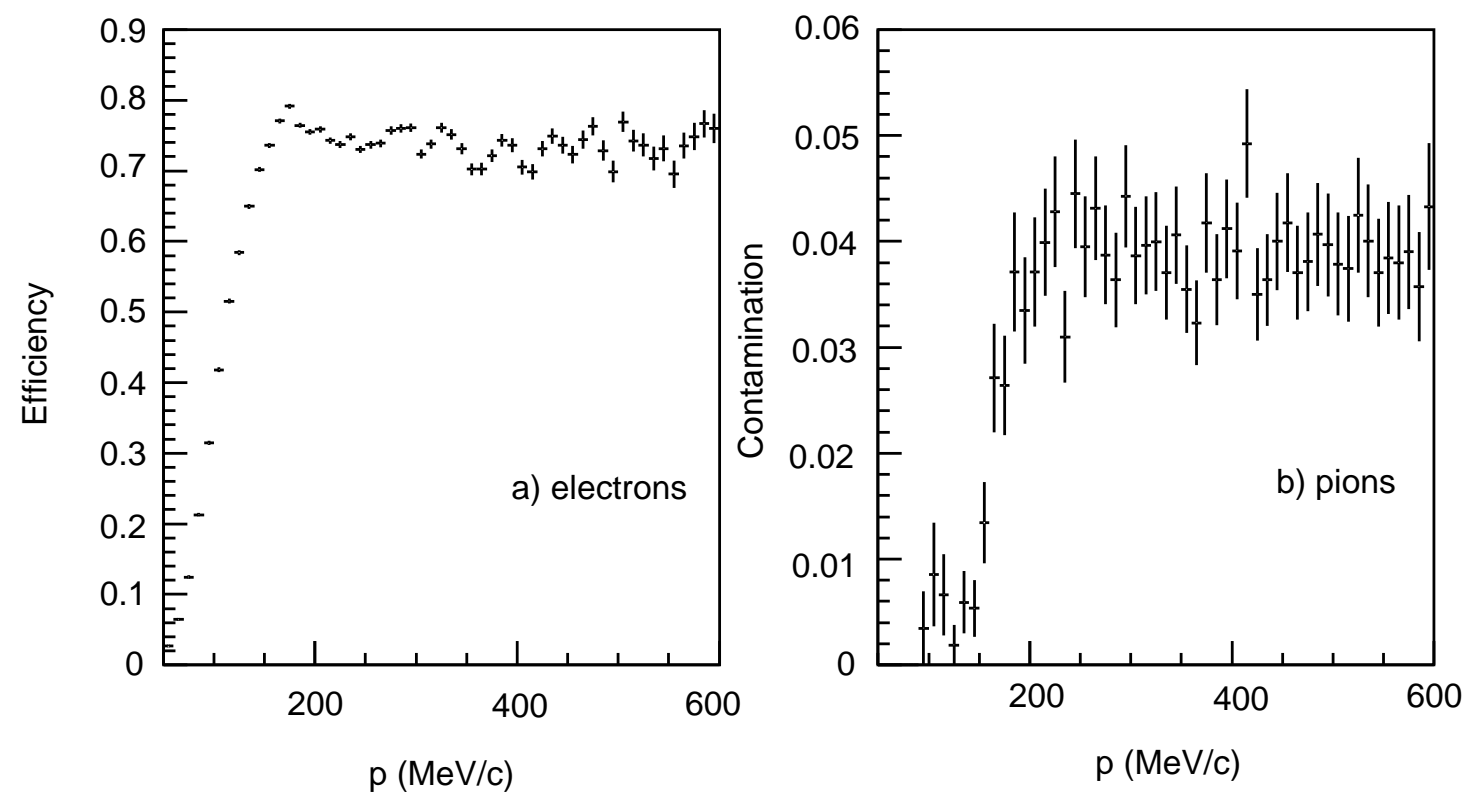

Figure 28: a) Electron identification efficiency, and b) pion contamination fraction as a function of $\mathrm{e} / \pi$ momentum.

\section{$5.9 \quad \mathrm{~K}^{0}, \overline{\mathrm{K}}^{0} \rightarrow \pi^{0} \pi^{0} \rightarrow 4 \gamma$ event reconstruction}

The transverse view together with the reconstruction of an $\overline{\mathrm{p}} \mathrm{p} \rightarrow \pi^{+} \mathrm{K}^{-} \mathrm{K}^{0} \rightarrow 2 \pi^{0} \rightarrow 4 \gamma$ event was displayed in Fig. 2. In order to determine the neutral-kaon decay time, the production vertex and the four-momentum of the neutral kaon are calculated from the track parameters of the charged kaon and pion, whereas the decay vertex of the neutral kaon along the $\mathrm{K}^{0}$ direction of flight is deduced from the photon showers measured in the ECAL. Four neutral showers need to be identified by the pattern recognition. These showers are not allowed to overlap in the W projection of the ECAL, ensuring the correct 
application of the photon energy calibration. To suppress photons generated by secondary interactions of charged tracks in the ECAL, only showers are accepted whose conversion point is separated by at least 50 or $25 \mathrm{~cm}$ from the charged kaon or pion track extrapolation into the ECAL, respectively.

The neutral-kaon decay time is determined by a full geometrical and kinematical reconstruction of the annihilation $\overline{\mathrm{p}} \mathrm{p} \rightarrow \mathrm{K}^{0} \mathrm{~K}^{-} \pi^{+}$and the neutral particle cascade $\mathrm{K}^{0} \rightarrow$ $\pi^{0} \pi^{0} \rightarrow 4 \gamma$ through a $6 \mathrm{C}$ fit. This constraint fit requires energy and momentum conservation, the equality of the missing mass at the annihilation vertex and the $\mathrm{K}^{0}$ mass, that the $\gamma \gamma$ invariant masses of the two $\gamma \gamma$ pairs are equal to the $\pi^{0}$ mass, and that the four photon flight directions intersect with the flight direction of the neutral kaon at one common point, namely the unknown neutral-kaon decay vertex [24, 25]. The fit provides a $\chi^{2}$-probability which is used for event selection.

As can be shown by simulation, the $\mathrm{K}^{0}$ momentum and the precise measurement of the photon conversion points contribute most of the information required to find the neutralkaon decay vertex. The photon energies are used in the constraint fit in order to find the correct association of the four photons to the two intermediate neutral pions. This is achieved by application of the constraint fit to all three possible $\gamma \gamma$ pairings. The pairing with the lowest $\chi^{2}$ value is chosen. The photon flight directions (shower angles) are not used in the constraint fit.

The constraint fit is applied with a photon conversion-point resolution of $\sigma_{r \varphi}=4 \mathrm{~mm}$ and $\sigma_{z}=5 \mathrm{~mm}$. Small variations of these resolutions, e.g. to account for photon conversions in the PID, do not significantly affect the neutral-kaon decay-time resolution. For the photon energy resolution, $\sigma_{w}(E)$ obtained by the photon energy calibration is used.

To demonstrate the ability of the constraint fit to identify and reconstruct the desired decay channel, a $4 \gamma\left(\pi^{0} \pi^{0}\right)$ and a $\gamma \gamma$ invariant-mass distribution are displayed in Fig. 29 for real data and MC events. The distributions are obtained by omitting the constraints
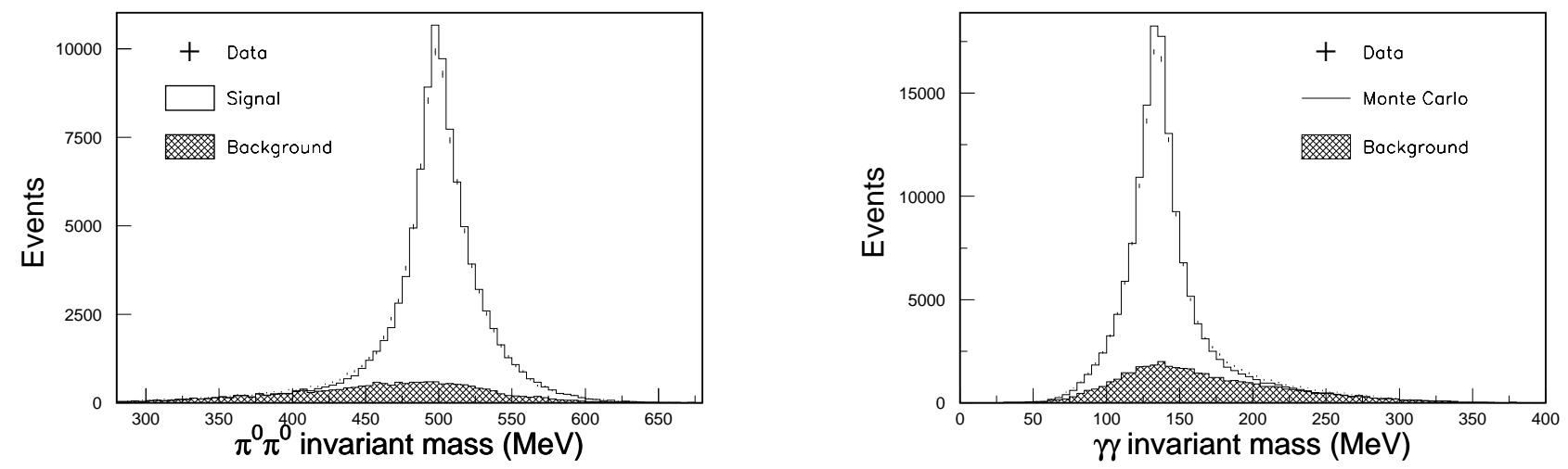

Figure 29: $4 \gamma$ and $\gamma \gamma$ invariant mass distributions obtained from constraint fits (see text). on the $\mathrm{K}^{0}$ mass or the $\pi^{0}$ masses in the constraint fit, respectively. The observed peak at 
the expected $\mathrm{K}^{0}$ mass has a resolution of $\sigma\left(m_{4 \gamma}\right) \approx 25 \mathrm{MeV} / c^{2}$; the peak at the $\pi^{0}$ mass has a resolution of $\sigma\left(m_{2 \gamma}\right) \approx 20 \mathrm{MeV} / c^{2}$.

The neutral-kaon decay length is reconstructed by the constraint fit with a precision of $2 \mathrm{~cm}$ (FWHM). This translates into a decay-time resolution of $0.7 \tau_{S}$ (FWHM) for the neutral kaon. In Ref. [5, 25], the implications of the achieved decay-time resolution on the $\mathrm{CP}$ asymmetry in $\mathrm{K}^{0}, \overline{\mathrm{K}}^{0} \rightarrow \pi^{0} \pi^{0}$ decays are discussed in detail, as well as additional analysis criteria. One of the additional analysis criteria is related to the use of the shower-angles $\phi^{\prime}$ and $\theta^{\prime}$, which allow an independent determination of the neutralkaon decay vertex. Along the neutral-kaon flight path - known from the $\mathrm{K}^{ \pm} \pi^{\mp}$ missing momentum and the annihilation vertex — one searches for the point with the minimal $\chi^{2}$ of the four shower-angle pairs. The rms resolution of this vertex is $5 \tau_{S}(15 \mathrm{~cm})$. This is of course much worse than the resolution of $0.7 \tau_{S}$ achieved with the standard constrained-fit method. Nevertheless, the vertex obtained from the shower angles is useful in this analysis for the following reason: The interference signal of the $\mathrm{K}^{0}, \overline{\mathrm{K}}^{0}$ decay asymmetry is pronounced at decay times of about $8 \tau_{S}$ and, therefore, well separated from annihilation background and $\mathrm{K}_{\mathrm{S}} \rightarrow \pi^{0} \pi^{0}$ events (predominantly at early decay times

of $\left.\leq 5 \tau_{S}\right)$. However, the tails of badly reconstructed events dampen the interference signal (preferentially short-lifetime events are shifted to longer decay times because of the exponential time dependence). Many of these events, accidentally reconstructed to be in the interference region, are recognized and rejected when the shower directions point to a decay vertex close to the annihilation vertex. This eliminates a sizeable fraction of the 'shifted' events from both $\mathrm{K}_{\mathrm{S}}$ decays, as well as annihilation background produced at zero decay time. In addition, the number of events with showers from secondary interactions of the primary tracks is reduced. As a result, the information from the shower angles does help to significantly increase the observed $\mathrm{K}^{0}, \overline{\mathrm{K}}^{0} \rightarrow \pi^{0} \pi^{0}$ asymmetry.

\section{Conclusions}

The design, construction, and performance of ECAL - the photon detector installed in the CPLEAR experiment - have been described. The physics of the CPLEAR experiment demanded a high position resolution (better than $5 \mathrm{~mm}$ ), high detection efficiency, and moderate energy resolution for photons of $\leq 700 \mathrm{MeV}$ energy. The performance of ECAL shows that the main criteria were achieved despite the space constraints imposed by the CPLEAR detector design, and the general problems of detecting low-energy photons. The operation was efficient and reliable, even though the modularity of the active part was not exploited as fully as foreseen.

The performance is, however, somewhat hampered in the actual environment of the CPLEAR experiment. In particular, the detection efficiency of photons (and electrons) below $100 \mathrm{MeV}$ is low, caused by the minimal requirements of the pattern recognition and by some $30 \%$ of the photons, which convert and deposit substantial energy in the detector elements in front of ECAL. The spatial resolution is also affected by photon conversion before the ECAL. Furthermore, the interaction and decay of charged particles from $\bar{p}$ annihilation produce a sizeable number of background photons, which are detected in ECAL alongside the photons from neutral-kaon decay. These photons cannot easily be identified and so render the analysis more difficult. In addition, a better measurement of the shower energy would be of help. 


\section{Acknowledgements}

We would like to thank the CERN LEAR staff for their support and cooperation. We acknowledge the Technical Support Group (G. Muratori and M. Price), the Electronics Development Group (H. Verweij and J.P. Vanuxem; F. Bourgeois and P. Pointing), the Wiring Pool (C. Millerin), the Drawing Office (W. Albrecht, P. Charra and D. Geiss), the Online Support Group (R. Divia, P. Rimmer, A. Vascotto), the Gas Service (H. Rigoni and D. Fromm).

The engineering advice of G. Kesseler and G. Petrucci was of great help. For the chambers, we benefitted from the ingenuity and skill of the late Max Renevey. For the strip plates, Prof. L. Piemontese kindly granted us access to the machine available in Ferrara, Italy and we gratefully acknowledge the work of Dr. V. Carassiti (Univ. Ferrara) and Mrs. G. Bonora (INFN Ferrara). We acknowledge the contribution by P. Bargassa, I. Mandic and M. Mikuz on the ECAL performance.

This work was supported by the Greek General Secretariat of Research and Technology and the Swiss National Science Foundation.

\section{References}

[1] R. Adler et al., The CPLEAR collaboration, Phys. Lett. B363 (1995) 237.

[2] R. Adler et al., The CPLEAR collaboration, Phys. Lett. B363 (1995) 243.

[3] R. Adler et al., The CPLEAR collaboration, Phys. Lett. B370 (1996) 167.

[4] R. Adler et al., The CPLEAR collaboration, Phys. Lett. B374 (1996) 313.

[5] R. Adler et al., The CPLEAR collaboration, Z. Phys. C70 (1996) 211.

[6] M. Wolter, Ph.D. thesis, ETH Zürich, 1996.

[7] P. Bloch et al., Nucl. Instrum. Methods A297 (1990) 126.

[8] R. Adler et al., The CPLEAR collaboration, Nucl. Instrum. Meth. A379 (1996) 76.

[9] R. Adler et al., Nucl. Instrum. Methods A321 (1992) 458.

[10] L. Piemontese, INFN and University of Ferrara, private communication.

[11] F. Bal et al., Nucl. Instrum. Methods A323 (1992) 511.

[12] E. Aslanides et al. in Proc. 8th Conf. on Computing in High Energy Physics, Santa Fe, NM 1990, AIP Conf. Proc. 209, eds. J.Illberg and M. Oothoudt, (AIP, New York, 1990) p. 589.

[13] F. Bal et al., Technical description of the F686F CRAM module, CERN ECP-EDA Internal Note, June 1991.

[14] Courtesy of B. Van Koningsveld, CERN.

[15] H. Müller et al., CERN Host Interface, user's manual, CERN/EP, 1989. 
[16] Courtesy of R. Divia, CERN.

[17] P. Kokkas, Ph.D. thesis, University of Ioannina, 1991.

[18] P.Bloch, CPLEAR Int. Rep. NFS 13, 1993.

[19] R. Adler, CPLEAR Int. Rep. NFS 2 and 5, 1993. R. Adler, Ph.D. thesis, University of Basle, 1994.

[20] G. Polivka, CPLEAR Int. Rep. NFS 6, 1993. G. Polivka, Ph.D. thesis, University of Basle, 1996.

[21] C. Felder, Ph.D. thesis, University of Basle, 1993.

[22] O. Behnke, Ph.D. thesis, ETH Zürich, 1996.

[23] The CPLEAR collaboration, Upper limit for the branching ratio of $\mathrm{K}_{\mathrm{S}} \rightarrow \mathrm{e}^{+} \mathrm{e}^{-}$decay, Nucl. Phys. A, Conf. Proceedings (LEAP'96), to be published.

[24] B. Pagels, Ph.D. thesis, University of Basle, 1992.

[25] B. Pagels, O. Behnke and H.-J. Gerber, Reconstruction of a particle decay containing intermediate decays of invisible neutrals, ETHZ-IPP Int. Rep. RP 95-04, 1995.

[26] B.R. Firth, Report on Industrial Placement, University of Bath/CERN Geneva, 1988.

[27] H.U. Johner, diploma thesis, University of Fribourg, Fribourg, 1987.

[28] The ALEPH Handbook 1989, CERN, Geneva, 1989.

[29] J.B. Lindsay, C. Millerin, J.C. Tarlé, H. Verweij and H. Wendler, Nucl. Instrum. Methods 156 (1978) 329.

[30] G. Muratori, A device for measuring the tension of wires, EP Int. Rep. 76-14, June 1976.

[31] R. Brun et al, GEANT 3 user's guide, CERN DD/EE/84-1, 1984.

[32] U. Mall, Ph.D. thesis, University of Basle, 1989.

\section{A Chamber Production}

\section{A.1 Construction layout}

Figure 30 shows an exploded view of a chamber. The construction and test of the chambers consisted of the following steps:

- Graphite coating

- Mechanical assembly

- Testing and conditioning (1) 


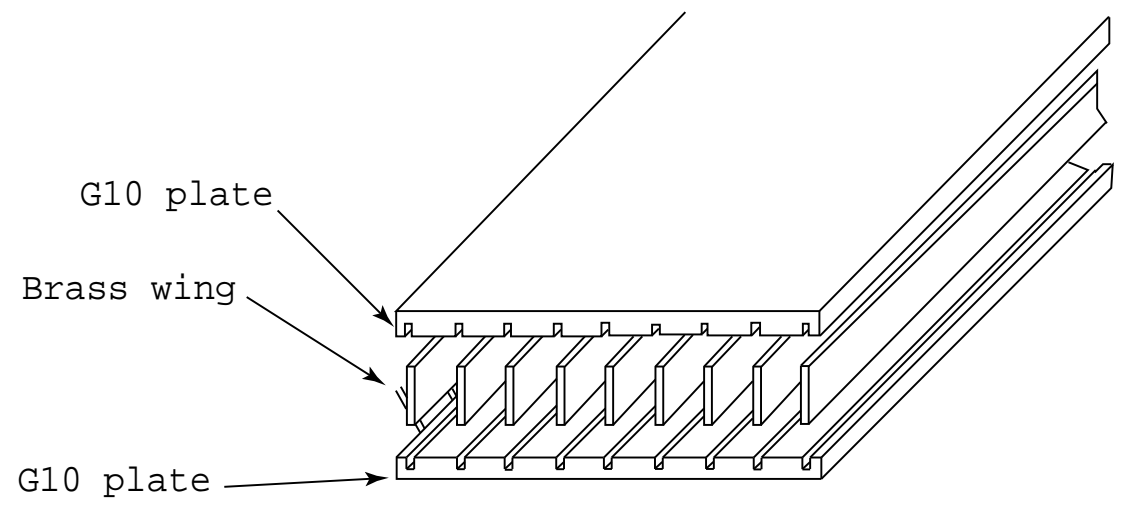

Figure 30: Exploded view of a chamber.

- Gas-tight sealing and conditioning (2)

- Wire tension measurement

The basic elements of the chambers are two G10 plates, $43 \mathrm{~mm}$ wide and $1 \mathrm{~mm}$ thick. In view of the insertion of metal wings, the plates were delivered with grooves $(0.5 \mathrm{~mm}$ deep, $1 \mathrm{~mm}$ wide). The plates were polished, and a good proportion of them was coated with a thin film on the side which was not grooved, to minimize the friction when positioning the chambers inside the ECAL. (This film did not affect the signals transmitted to the adjacent strips.) Once at CERN, the plates were cleaned with trichlorethylene.

\section{A.2 Graphite coating}

As explained in Ref. [7], the grooved side of each plate was coated with a solution containing graphite ${ }^{19}$, chosen such that the quality of the coating did not deteriorate when immersed in pentane and adjusted to give a resistance in the $1 \mathrm{M} \Omega / \square$ region. This resistance was chosen as a compromise between two conflicting requirements, namely: high resistance for high pulse-heights on the strips, and low resistance to ensure good charge collection at high intensities [7]. The plates were scored on a board in two sets of 25 , the longer side parallel to the floor. The graphite was then sprayed onto the plates from a distance of about $1 \mathrm{~m}$ using a car sprayer moving horizontally. On either end of the board, $33 \mathrm{~mm}$ were left untouched.

Resistance measurements were performed after a drying period of $24 \mathrm{~h}$. A significant decrease in the resistance was observed during the drying period, the length of which is strongly dependent on the humidity. The resistance was measured with a custom-made instrument [26], designed to test simultaneously the eight paths between the grooves of a chamber plate. This measurement was done at 5 different points equally spaced along the plate. The uniformity between different points on the same plate was better than $20 \%$ for most of the plates. We accepted the plates when the resistance $R$ was $0.5 \leq R \leq 2.5$ $\mathrm{M} \Omega / \square$. Less than $10 \%$ were discarded. Those with too high a resistivity value required polishing with a piece of cloth in order to be accepted. The effect of this operation was probably to establish better contacts between the graphite grains of the solution used.

\footnotetext{
${ }^{19}$ G33 mit verminderter Leitfähigkeit, Kontakt Chemie, 76437 Rastatt, Germany. (The solution was specially prepared to fit our requirements.)
} 


\section{A.3 Mechanical assembly}

A custom-made grooved aluminium jig (Fig. 31) was used to glue a set of nine brass wings fitted with seven support bridges to one of the two G10 plates. A conductive glue made of Araldite and graphite powder was prepared for each set of 25 chambers, taking $2.3 \mathrm{~g}$ of resin and $1.0 \mathrm{~g}$ of graphite, then adding the hardener and mixing. Resistivity measurements with various mixtures of Araldite, hardener, and graphite powder, as well as different graphite grain sizes, were performed to find the best compromise between resistivity, viscosity, drying time, and cost [27].

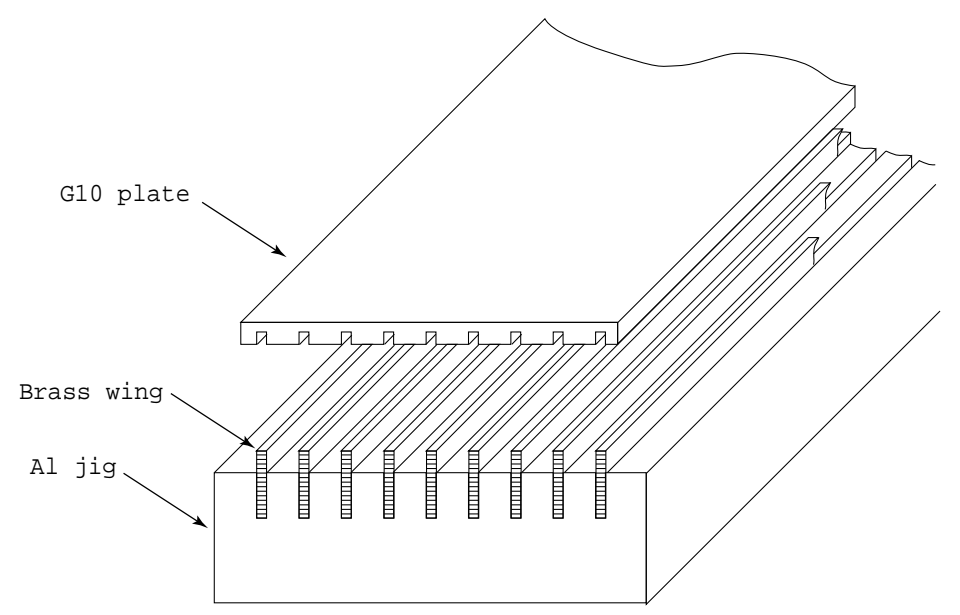

Figure 31: Jig for glueing the brass wings.

\section{A.3.1 Loading the glueing jig}

- The grooves were carefully cleaned, making use of compressed air and a scraper.

- The brass wings $\left(5 \times 1 \mathrm{~mm}^{2}\right.$ cross-section and $2700 \mathrm{~mm}$ length) were cleaned with chloroethene and then inserted in the aluminium jig. The upper side of each wing was $1.5_{-0.0}^{+0.1} \mathrm{~mm}$ from the upper plane of the jig. This distance was measured at a number of points along the wing. The seven slits of $0.2 \mathrm{~mm}$ width and $1 \mathrm{~mm}$ depth (cut along the wing every $35 \mathrm{~cm}$ by an external firm) stay on the outside of the jig.

The support bridges were inserted into each slit using a special tool . The top of the bridge was about $0.7 \mathrm{~mm}$ above the top of the wing. These bridges (Fig. 32) are very similar to those used in Ref. [28].

- The graphited glue was collected on a plastic roller which was then rolled over the brass wings.

- Immediately afterwards one of the G10 plates was carefully laid over the jig, the nine wings fitting into its grooves.

The 25 jigs so loaded were then laid inside a closed vessel where they were kept at a temperature of $40^{\circ} \mathrm{C}$ for about 24 hours, typically overnight following the first day of the construction period. 


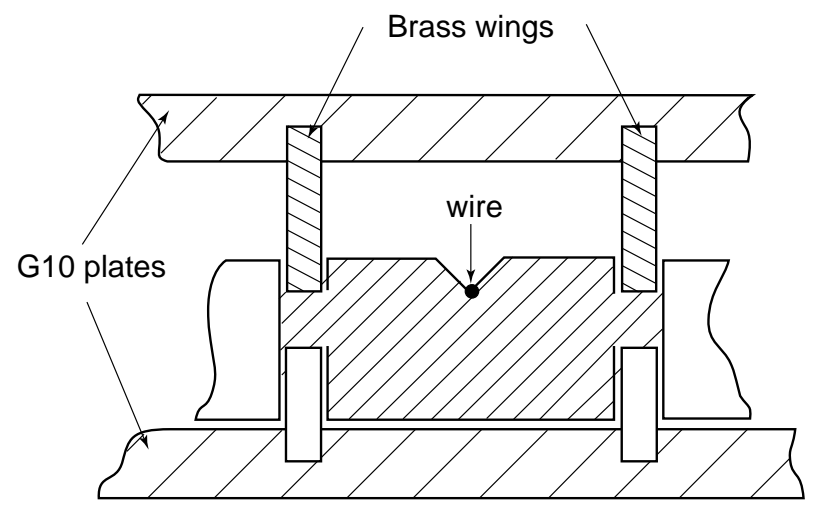

Figure 32: Plastic bridge to support the wires.

\section{A.3.2 Completing the cradle}

Once out of the vessel, the profile (cradle) formed by the G10 plate and the wings was freed from the jig and prepared for further equipping. The resistance between any two adjacent wings was measured. A value larger than a few $\mathrm{M} \Omega$ was taken as an indication that the glueing was not good. About $1 \%$ of the cradles were discarded for this reason. This selection guaranteed a good charge collection out of the resistive cathode, which was necessary because of the high rate of ionizing particles expected per wire.

The nine brass wings were then electrically connected by hard soldering them at either end to a $5 \mathrm{~mm}$ wide and $0.5 \mathrm{~mm}$ thick brass strip. On either end, a printed-circuit board (Fig. 6) machined to accommodate the $0.5 \mathrm{~mm}$ thick strip was then laid over the brass strip and glued with slow Araldite. The glue was left to dry overnight.

The gas supply is serial through the chambers of a layer, i.e. the input of a chamber is connected to the output of the previous one by a small stainless-steel pipe. One of the difficulties was to obtain a gas feedthrough which would not exceed the chamber thickness $(6 \mathrm{~mm})$ and would not be damaged by pentane vapours. We finally decided to use modified Lemo ${ }^{20}$ plugs which had been milled for the insertion of a small O-ring.

\section{A.3.3 Wire stretching}

On the third day, the 25 cradles were transferred to another working surface, where a frame on which $25 \times 8$ wires had been prestretched with the proper mechanical tension (1.60 $\mathrm{N})$ was fitted on the cradles. The wires were soldered one by one on the printed-circuit boards on either side of the cradle. The boards were then cleaned with trichlorethylene, and covered with Araldite, both for mechanical and insulation purposes. After drying again overnight, on the morning of the fourth day the wires were secured with a drop of glue ${ }^{21}$ to each of the seven bridges to avoid later mechanical instabilities. The glue over each bridge was dried with a UV lamp for $12 \mathrm{~min}$.

On the following morning, further cleaning of the printed-circuit boards was performed as well as the important operation of cleaning the wires. In both cases trichlorethylene was used. The 25 chambers were then ready for the first HV tests.

\footnotetext{
${ }^{20}$ W.W.Fischer, 1143 Apples, VD, Switzerland.

${ }^{21}$ NORLAND/UV123, EPOTECHNY, 78140 Velizy-Villacoublay, France.
} 


\section{A.4 Testing and conditioning (1)}

'Plateau' curves measured on prototype chambers had shown that the full detection efficiency was reached at a HV slightly above $3000 \mathrm{~V}$. The conditioning procedure aimed to prepare the chambers to stand a $\mathrm{HV}$ of $3400 \mathrm{~V}$ (corresponding to an electric field of 350 $\mathrm{kV} / \mathrm{cm}$ at the surface of the anode wire) without appreciable noise.

One of our problems for the conditioning of the chambers was the presence of gold flakes on the anode wires (see Fig. 33). We pulled the wire between two layers of clean

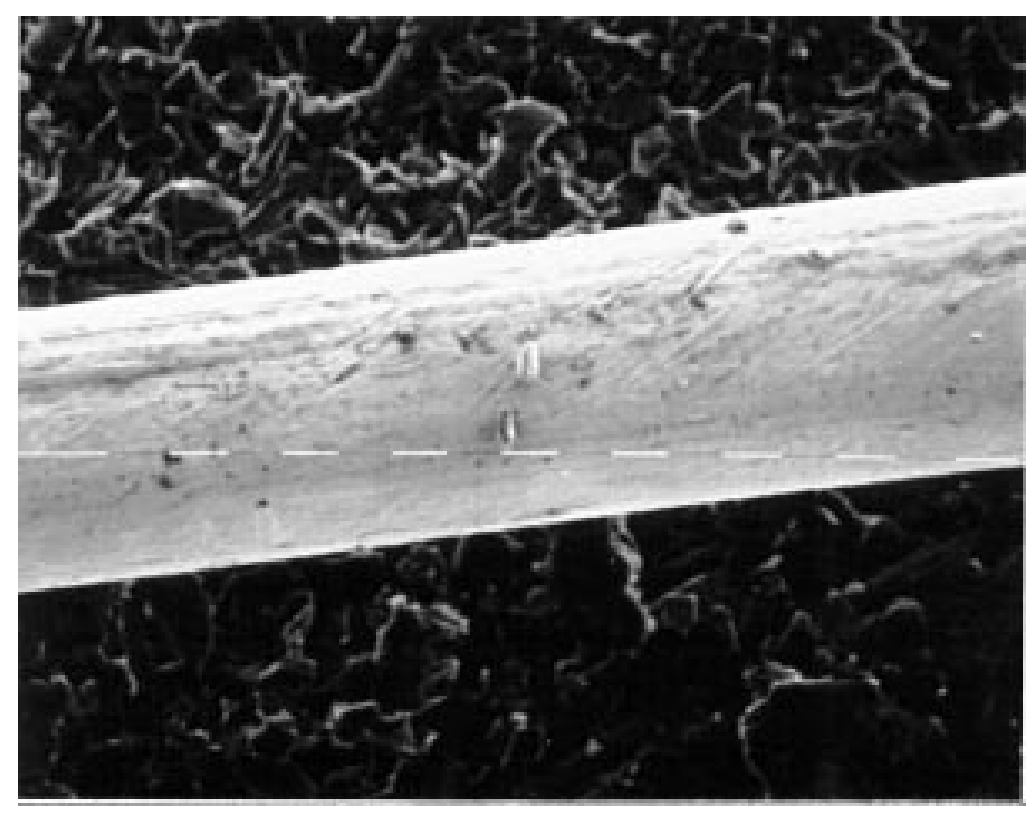

Figure 33: Gold flakes on the anode wires.

paper and noticed that it tended to leave a slight deposit which, under the microscope, appeared to consist of tiny flakes of gold. These were responsible for a dark current as high as a few $\mu \mathrm{A}$ at a $\mathrm{HV}$ as low as $2200 \mathrm{~V}$ in air. Therefore the first conditioning operation was to get rid of these flakes. We had no success in brushing and cleaning the wires with solvents while they were passing through a stretching machine. An improvement was obtained by taking the wires between rubber-tipped tweezers and sliding them very gently along the wires. Then, a current measurement was performed for each individual wire in air with a $\mathrm{HV}$ of $2200 \mathrm{~V}$. The rubbing operation was repeated until the current was $<1 \mu \mathrm{A}$.

Another problem was encountered when raising the HV. Even though the current was carefully checked, strong discharges occurred suddenly, causing the wire to break. Therefore, we decided to use a more sensitive method to establish the rate of HV increase by relying on the rate of microdischarges recorded in an RMH system [29] with one channel per wire.

A test bench was installed to condition 78 chambers independently. An FIC8230 was used to analyse the rates from the 624 wires and to drive, according to these rates, the 78 independent HV channels from two CAEN HV power supplies. Such cycles of rate counting and HV adjustments were repeated every other minute.

The chambers were arranged in three groups of 26. Two groups were equipped with gas-tight containers in which chambers could be installed with their covers only pressed in 
place (not yet glued, so that they could still be opened for further cleaning). The chambers in the containers as well as the closed chambers in the third group were supplied by gas (air, $\mathrm{CO}_{2}$, or a $\mathrm{CO}_{2}$ /pentane mixture) in series of 12 or 14 .

Once the chambers had been installed in the container, the conditioning started with air at a HV of $500 \mathrm{~V}$. After a one-minute counting cycle, the HV was either increased by $100 \mathrm{~V}$ if the total counting rate of the chamber was less than $1 \mathrm{kHz}$, decreased by 200 $\mathrm{V}$ if it was larger than $2 \mathrm{kHz}$, or left unchanged otherwise. This procedure was repeated until a HV of $2000 \mathrm{~V}$ was reached. A final increase to $2100 \mathrm{~V}$ was only achieved when the counting rate had decreased to below $40 \mathrm{~Hz}$. The conditioning in air was considered satisfactory when this limit of $40 \mathrm{~Hz}$ was maintained for more than 10 consecutive cycles. The conditioning process lasted between one and three hours.

Afterwards, the chambers were rinsed with $\mathrm{CO}_{2}$ (for safety reasons) for $15 \mathrm{~min}$, then with the $\mathrm{CO}_{2}$ /pentane gas mixture for 90 min before turning on the $\mathrm{HV}$ in gas.

The conditioning process with the $\mathrm{CO}_{2}$ /pentane gas mixture started at a $\mathrm{HV}$ of 2000 $\mathrm{V}$. After one-minute counting cycles, the HV was increased by $100 \mathrm{~V}$ when the counting rate was $\leq 1 \mathrm{kHz}$. When the counting rate was $\geq 1 \mathrm{kHz}$, the $\mathrm{HV}$ was decreased by 200 V. From 3000 to $3400 \mathrm{~V}$, the HV was increased if the counting rate had been smaller than $300 \mathrm{~Hz}$ for 3 consecutive cycles, decreased by $200 \mathrm{~V}$ if it was larger than $500 \mathrm{~Hz}$, and left unchanged otherwise. When $3400 \mathrm{~V}$ were reached and the counting rate had decreased below $100 \mathrm{~Hz}$ for more than 10 consecutive cycles a plateau curve was recorded. It consisted of 10 consecutive counting cycles, each performed at $10 \mathrm{HV}$ settings ranging from 2500 to $3400 \mathrm{~V}$ in steps of $100 \mathrm{~V}$. This first conditioning process was considered satisfactory if the counting rate was always below $100 \mathrm{~Hz}$. Those chambers passing the conditioning successfully were set to a 'stand-by' HV of $3200 \mathrm{~V}$ until the other chambers of the group were ready, but were still monitored by counting the rates. This process typically lasted for about a day, but in some cases for up to a week.

During all this conditioning, the overcurrent trip feature provided by the CAEN power supplies was used for protection against a sudden rise in the current. As soon as the current had reached $1 \mu \mathrm{A}$ the HV was automatically decreased by the power supply so that this maximum allowed current would not be exceeded. If this situation lasted for more than $10 \mathrm{~s}$, the HV was automatically turned off. When the control program detected a 'trip' situation for a chamber, the HV value was decreased by $200 \mathrm{~V}$ and the HV was turned on again unless a trip had happened four times in less than $30 \mathrm{~min}$.

This automatic conditioning procedure allowed us to cope with the 25 chambers that were built every day. Out of the 2500 chambers that have been built only 5 could not complete the conditioning process with success.

\section{A.5 Gas-tight sealing and conditioning (2)}

The cover - up to now just laid over the cradle — was secured with a mixture of Ay103 resin and Hy991 hardener in the proportion 1.0 : 0.4 . This mixture was stirred carefully for about 30 min until it became transparent, and injected into the enclosures between the top and bottom covers using a syringe. The quantity of glue needed was about $0.6 \mathrm{~g}$ per chamber. After a $12 \mathrm{~h}$ drying period, the chambers were put back into the containers and connected in series to the gas supply to go through a second conditioning cycle, which typically lasted several hours. During the various manipulations, however, a short circuit occasionally occurred in one of the eight channels. At this stage only part of the chamber 
could be saved by cutting away the pin connecting the wire to the HV supply. Some of the chambers were opened for a close inspection; a broken wire was found in all cases. The breaking of the wires could not be traced back to a possible 'weak' point of the mechanical structure, such as, for instance, the soldered points over the printed-circuit boards on both sides of the chamber, or the glued points on the bridges.

All the chambers surviving the second conditioning were checked against gas leaks. The chambers were filled with $\mathrm{CO}_{2}$ gas at an overpressure of 18 mbar and monitored for about $24 \mathrm{~h}$. Some of the chambers were leaking around the caps. Additional Araldyte easily cured the problem.

\section{A.6 Wire tension measurement}

For a sample of 25 chambers (one every 100 chambers produced), the wire tension was tested at five points along each wire (between bridges 1 and 2, 2 and 3, 3 and 4, 4 and $5,5$ and 6$)$. The wire tension is constrained by two limits. The lower limit is given by the need to avoid oscillations of the wire leading to a break. The upper limit is defined by the permanent deformation of the wire. For our geometry and an $\mathrm{HV}$ of $3.5 \mathrm{kV}$, we find that the tension should be above $0.13 \mathrm{~N}$, and that the elastic constant of the wire requires a tension below $2.6 \mathrm{~N}$. The tension $T$ of a wire of length $L$ and specific mass $\mu$ can be determined [30] by measuring the frequency $\nu$ of vibration inside a magnetic field: $T=4 L^{2} \mu \nu^{2}$. The average frequency of the first harmonic is $380 \pm 50 \mathrm{~Hz}$, giving an average tension of $1.5 \mathrm{~N}$ with $L=0.35 \mathrm{~m}$ and $\mu=24.1 \times 10^{6} \mathrm{~kg} / \mathrm{m}$. No chamber had to be discarded for an unsatisfactory value of the wire tension.

\section{B Monte Carlo simulation for ECAL}

The MC simulation is important for understanding the conversion point resolution, where the simulated data facilitate the assessment of the performance figures which otherwise would have been very difficult to achieve without dedicated particle beams. MC-simulated data are also an inevitable ingredient in the physics analysis for the reconstruction of the neutral decay vertices $\mathrm{K}^{0} \rightarrow \pi^{0} \pi^{0} \rightarrow 4 \gamma$ and $\mathrm{K}^{0} \rightarrow \pi^{0} \pi^{0} \pi^{0} \rightarrow 6 \gamma$. For the simulation of the ECAL, it is crucial to understand and simulate properly the wire and strip response to minimum-ionizing particles crossing the calorimeter.

Electromagnetic showers are simulated using the GEANT package [31]) with a cutoff energy of $400 \mathrm{keV}$ (the dependence on the cut-off parameter was studied over a wide range of energies from 10 to $700 \mathrm{keV}$; below $400 \mathrm{keV}$ the wire efficiency varied by less than $5 \%[32]))$. Since the tubes are operated in the limited streamer mode, the response of the wires (tubes) is assumed to be independent of the particle energy, as is the response of the strips. Therefore, the response of the calorimeter to showers can be simulated assuming that each of the $\mathrm{e}^{+}$or $\mathrm{e}^{-}$tracks gives a calorimeter response very similar to minimum-ionizing pions or muons.

The input for tuning the MC includes the efficiencies and hit multiplicities of the wires and the strips, as well as the noise of the strips (reported in Section 5.1). The numbers used are averages and do not take full account of the gradual increase of the number of dead chambers. The study for modelling and tuning the response of the ECAL in the simulation is based on minimum-ionizing pions penetrating the ECAL to the last layers. 
For the particles crossing the edges and wings of the tubes, a minimal track distance of 1 $\mathrm{mm}$ is required inside the tube in order to set the 'hit'.

Wires: We introduced a wire inefficiency of $12 \%$ into the simulation (this inefficiency was only $4 \%$ for the prototype operating at $3.2 \mathrm{kV}[9])$. For the higher hit multiplicities $(m \geq 2)$ the spatial distribution was analysed. For $m=2$, the two hits were adjacent in $80 \%$ of the cases.

Model for the $M C$ simulation of the strip response: To reproduce the strip multiplicity and strip efficiency, we relied on the measured charge distribution (peak position corresponds to one charge unit) and the spatial distribution of the induced charge published in $[7]$.

For the crossing of a minimum-ionizing particle in a tube, the induced charge is generated according to this charge distribution and, depending on the position relative to the strip centre, distributed on the central strip and its four neighbouring $( \pm 2)$ strips, following the spatial distribution. A threshold is then applied on each strip. Depending on this threshold value, both strip multiplicity $(0 \leq m \leq 5)$ and efficiency are obtained for each position. For a low threshold value of 0.2 (charge units), very good agreement is achieved with the prototype measurement (efficiency: 98\%, strip multiplicity: 1.5). For a higher threshold ( 0.35 charge units), the inefficiency is $23 \%$ and the probability of multiplicity $m=2$ is $11 \%$, in very good agreement with the real data.

In summary, knowing the inefficiency of a strip layer in a given spatial region of the calorimeter, a threshold value which corresponds to this inefficiency can be chosen. The relationship between inefficiency and threshold can be easily parametrized. Then, for every track crossing the layer in this region, the charge is generated according to the following recipe: share it among the neighbouring strips and apply the threshold to obtain the strip multiplicity.

Simulation of the noise in the strips: The presence of the noise in the strips was mentioned in Section 5. For the simulation, the noise is parametrized in terms of the cluster size, the number of clusters, and the position. The noise is added randomly to the hits set by the tracking of charged particles. The number of noise clusters is parametrized with an exponential. The cluster size is parametrized with two exponentials. The position of the noise in the layer follows a Gaussian distribution (width $\approx 50$ strips), centred on the longest strips. A flat term is added for selected layers if necessary. 